Article

\title{
Accurate Fall Detection and Localization for Elderly People Based on Neural Network and Energy-Efficient Wireless Sensor Network
}

\author{
Sadik Kamel Gharghan ${ }^{1, *} \mathbb{C}$, Saleem Latteef Mohammed ${ }^{1}$, Ali Al-Naji ${ }^{1,2}$, \\ Mahmood Jawad Abu-AlShaeer ${ }^{3}$, Haider Mahmood Jawad ${ }^{4}$, Aqeel Mahmood Jawad ${ }^{4}$ and \\ Javaan Chahl ${ }^{2,5}$ \\ 1 Department of Medical Instrumentation Techniques Engineering, Electrical Engineering Technical College, \\ Middle Technical University, Baghdad 10013, Iraq; salimlatif62@yahoo.com (S.L.M.); \\ ali_abdulelah_noori.al-naji@mymail.unisa.edu.au (A.A.-N.) \\ 2 School of Engineering, University of South Australia, Mawson Lakes, SA 5095, Australia; \\ Javaan.Chahl@unisa.edu.au \\ 3 Department of Statistic, Al-Rafidain University College, Baghdad 10064, Iraq; mahmoud_juad@yahoo.com \\ 4 Department of Computer Communication Engineering, Al-Rafidain University College, \\ Baghdad 10064, Iraq; haider_mahmood2003@yahoo.com (H.M.J.); \\ aqeel_mahmood_1986@yahoo.com (A.M.J.) \\ 5 Joint and Operations Analysis Division, Defence Science and Technology Group, \\ Melbourne, VIC 3207, Australia \\ * Correspondence: sadiq_gharghan@yahoo.com; Tel.: +964-773-639-3936
}

Received: 5 October 2018; Accepted: 19 October 2018; Published: 23 October 2018

check for updates

\begin{abstract}
Falls are the main source of injury for elderly patients with epilepsy and Parkinson's disease. Elderly people who carry battery powered health monitoring systems can move unhindered from one place to another according to their activities, thus improving their quality of life. This paper aims to detect when an elderly individual falls and to provide accurate location of the incident while the individual is moving in indoor environments such as in houses, medical health care centers, and hospitals. Fall detection is accurately determined based on a proposed sensor-based fall detection algorithm, whereas the localization of the elderly person is determined based on an artificial neural network (ANN). In addition, the power consumption of the fall detection system (FDS) is minimized based on a data-driven algorithm. Results show that an elderly fall can be detected with accuracy levels of $100 \%$ and $92.5 \%$ for line-of-sight (LOS) and non-line-of-sight (NLOS) environments, respectively. In addition, elderly indoor localization error is improved with a mean absolute error of 0.0094 and $0.0454 \mathrm{~m}$ for LOS and NLOS, respectively, after the application of the ANN optimization technique. Moreover, the battery life of the FDS is improved relative to conventional implementation due to reduced computational effort. The proposed FDS outperforms existing systems in terms of fall detection accuracy, localization errors, and power consumption.
\end{abstract}

Keywords: accelerometer sensor; data-driven algorithm; fall detection; localization; neural network; tilt sensor; wireless sensor network (WSN); ZigBee

\section{Introduction}

The worldwide population of elderly who are more than 65 years old is expected to grow to 1 billion in 2030, and the percentage of individuals aged 20-64 years will become 35\% of the population [1]. Therefore, monitoring the vital signs of elderly people is essential. In vital sign observation applications, patients and elderly people wear sensors that manage their vital parameters 
to detect emergency conditions and enable caregivers to respond efficiently. A fall is one of the key factors that can lead to injuries and decrease quality of life, at times resulting in the death of elderly persons. People's rate of falling increases with their age. Falls occur frequently in medical health care centers, hospitals, or houses, with approximately $30 \%$ of falls causing injury. Falls in hospitals occur in the rooms of the patients $(84 \%)$ and during transfer from one place to another $(19 \%)$. Furthermore, the majority of falls occur in areas adjacent to chairs and beds [2]. Most people who experience falls need special care in a nursing home or hospital, thereby restricting their life activities. The hazard issues of fall or slight fall, especially of the elderly, can be aggravated by chronic diseases, such as osteoporosis, delirium, and dementia [3]. The degree of danger from a fall for aging persons is frequently decided by the location of the fall, time of fall detection, duration and time of transfer and rescue services. Therefore, automatic detection of elderly people's falls along with the locations of the incident is important so that medical rescue staff can be dispatched immediately and so that the family of the elderly can be informed about the incident through a specific wireless network or mobile telephone.

The development of microelectromechanical technologies allows the integration of different sensors, and a wireless network is commonly used. Wireless sensor networks (WSNs) comprise a number of tiny and small sensor nodes which are deployed over several applications to monitor the physical environment (e.g., temperature, humidity, vibration, pressure, etc.) [4], patients vital signs (e.g., heart rate, body temperature, blood pressure, diabetes, motion, fall detection, etc.) [5], fog-supported smart city [6], fog-supported peer-to-peer WSN [7], etc. WSN have played a significant role in medical applications for monitoring elderly patients' vital signs. Physiological parameters can be monitored in the elderly to evaluate their vital signs, such as blood pressure and sugar, heart functions, postures, fall, and the location of a fall incident. Among these parameters, fall detection and localization are considered in this study. The power consumption problem of the proposed fall detection system (FDS) is also addressed. Several fall detection and localization systems have been developed recently. Fall detection can be categorized into three systems, namely, (i) vision-based, (ii) ambient sensor-based, and (iii) wearable sensor-based [8]. Vision-based systems employ a computer to capture images and videos of fall events. This method can be subdivided into three types: single RGB camera, 3D-based methods utilizing depth cameras, and 3D-based methods employing several cameras [9]. This system monitors the shape and position of the subjects, which depends on image processing preprocessing and pattern recognition techniques. Although this system is convenient and non-obtrusive for elders, it is more expansive than the other two types because it needs many cameras. In addition, the RGB camera needs to be calibrated to allow a 3D reconstruction of the body, resulting in a time-consuming and computationally intensive procedure [10].

Ambient sensor-based systems utilize external sensors such as acoustic, electromyography, vibration, infrared, and pressure sensors. The sensors of the ambient sensor-based methods are used in the area around the subject of concern. Sensors are implanted in the surroundings and track the movements of the elderly. Pressure sensors are usually employed in this method because they are unobtrusive, low cost, and sensitive. The main shortcomings of these methods are low fall detection accuracy (less than 90\%) [11], a tendency to generate false alarms when pressure sensors are used because the sensor senses the objects around the body, and the direct effect of distance between the body and sensor location on fall detection accuracy. Indoor environments should contain several sensors to obtain high accuracy, thereby increasing costs. With the development of microelectromechanical technologies in recent years, wearable sensor-based systems have become commonly used for fall detection. The sensors of the last method are attached to the subject of interest. The wearable sensor-based method usually depends on accelerometer sensors, which are connected to the body and provide an extraordinary level of obtrusiveness [8]. Accelerometers are popular wearable sensors embedded in FDSs to detect the position and movement of the subject [12]. The advantages of wearable accelerometer sensors are their small size, lower cost than external sensors, ability to be easily carried by the body [13], and ability to measure acceleration in three coordinates or angles of incidence. Thus, 
among the above three methods, the wearable sensor-based method using accelerometer sensors is considered in this study.

In elderly indoor localization, accurate estimation of node locations poses a challenge. One of the prevailing methods is a WSN integrated with several sensors to detect and localize the patient's fall. Localization and power consumption are essential challenges in WSN, where elderly people who carry a battery power system may transfer from one place to another according to their activities. Thus, a localization technique that can independently measure or determine the location of the elderly is necessary. Various range-based and range-free localization techniques are employed in WSN localization. Range-based techniques are employed to identify the distances and angles among nodes in WSN. Some examples of range-based localization techniques are angle of arrival (AoA), time difference of arrival (TDoA), time of arrival (ToA) [14], received signal strength indicator (RSSI) [15], global positioning system (GPS) [16], and acoustic energy [17]. Conversely, range-free localization techniques are less accurate than range-based localization and are less cost effective. They rely on the transmission link among mobile nodes and beacon nodes (called beacons nodes) in the network to determine node positions, and they do not provide information about distance and angle. In this study, a sensor node or FDS is carried by elderly people with restricted energy sources and minimal equipment requirement. Range-free localization techniques that depend on received signal strength indicator (RSSI) measurements are adopted because they do not require additional hardware, thereby minimizing power consumption, cost, size, and system complexity.

In this paper, the fall and location of subjects are determined by an FDS wearable device. The FDS, which includes a digital tilt sensor, accelerometer sensor, shock sensor, microcontroller, Zigbee wireless protocol, and power unit, is wrapped around the waist of the subject. The waist is selected as the location because it allows high discrimination between activities with the accelerometer system [18]. Digital tilt, shock, and accelerometer sensors are utilized to detect the fall incident of the subject. The location of the incident is estimated by using the RSSI of three Zigbee beacons for line-of-sight (LOS) and non-line-of-sight (NLOS) indoor environments. The RSSI of the three Zigbee beacons is used because no additional hardware is required [19]. The beacons are used to determine the location of the subject (i.e., location of fall incident) based on an artificial neural network (ANN) technique. Location accuracy is a crucial factor for WSN applications [20] because it can reduce the power consumption of the WSN nodes. When the location or distance among the WSN nodes is measured accurately, the transmitted power of the sensor node's transceiver radio module can be modulated to reduce power consumption, while still retaining connectivity, thereby prolonging battery life [21]. However, this approach is not considered in the current work. The fall alarm and raw RSSI data are sent to the coordinator node $(\mathrm{CN})$ for elderly location estimation and immediate rescue. Thus, the survival probability of elderly people who have fallen can be increased, with a population effect of increased life expectancy.

Practical wearable devices carried by patients, elderly people, and athletes are battery powered. Thus, the power consumption of these devices must be considered. The radio frequency (RF) element generally consumes the most power in a wearable device [22]. Several studies on wearable telehealth and telecare systems state that their suggested systems were energy efficient. Most of these studies employed only low-power hardware, adopting a low-power communication module or a microcontroller. However, they neither considered power reduction techniques to improve the power consumption of their systems nor focused on realizing full system functionality. Accordingly, the power consumption of the sensor node is improved in the present study with the use of a data-driven algorithm (DDA) along with a low-power wireless communication module (i.e., Zigbee) and a standalone microcontroller. With the use of low-power components and DDA, the FDS battery lifetime is extended. In DDA, the FDS can enter sleep mode when no information of an elderly fall (i.e., the normal case) is ready for transmission to the $\mathrm{CN}$. Once a fall happens, the sensor node wakes up for a short time to send the fall alarm and location information (i.e., RSSI values) to the $\mathrm{CN}$ and 
then returns to sleep mode to conserve energy. Instead of continuously monitoring the patients' vital signs, the FDS transmits data at sparse events.

The contributions of this paper are as follows:

1. Fall detection of elderly people is accurately detected based on FDS carried by the patient

2. The location of the fall is determined with minimum localization error based on ANN technique in indoor environments for LOS and NLOS scenarios.

3. The power consumption of the FDS carried by the subject is minimized based on DDA.

4. Fall detection accuracy achieved using sensor-based fall detection algorithm (S-BFDA), localization error based on ANN technique, and FDS power consumption based on DDA are compared with those of state-of-the-art systems.

\section{Related Works}

Different approaches or schemes have been used to determine or detect the fall of the elderly considering several application scenarios. Three categories of elderly FDSs are available, as highlighted in the introduction. Wearable sensor-based approaches have an advantage over the other two approaches because such approaches involve attaching the device to the subject which then moves with them. Such systems need sensors such as gyroscopes and accelerometers. Smartphones can be efficiently employed for elderly fall detection because most mobile smartphones include these sensors [23]. Many studies on fall detection applications show the applicability of Zigbee or Bluetooth radio solutions in wearable wireless sensor devices. One study [3] designed and implemented a care system that can detect and localize the fall of a human. The proposed system architecture consisted of a portable device such as a mobile phone or a tablet, the Zigbee wireless protocol as an access point, a console, a server, and an end-user mobile phone. The portable device automatically detects the fall of a subject and sends an alert message to the nursing center via the Zigbee access point. The proposed system achieved an average fall detection accuracy of $99.9 \%$. In addition, the system could determine the location of the fall incident based on the triangulation method to help medical staff find the patient quickly. The proposed system was efficient in terms of fall accuracy and power consumption, as the system could work continuously for 1 month without the need for a battery change and could send fall and location information more than 500 times.

In Reference [24], the author proposed a wireless body area network (WBAN) for fall detection applications and optimized the energy efficiency and throughput of the Zigbee communication protocol. A fall detection algorithm was implemented based on an accelerometer sensor worn on the subject's waist and could detect eight different fall cases. Results demonstrated that $100 \%$ sensitivity and specificity could be expected when the fall occurred in the vertical position (i.e., falls sliding against a wall ending in vertical position). The power consumption of the WBAN was minimized and was 10 times less based on the sleep/wake mode of the Zigbee, which transmitted data every $5 \mathrm{~s}$ and slept the rest of the time. In Reference [25], an outdoor healthcare monitoring system that could monitor electrocardiogram (ECG) signal and fall events of elderly people was designed and implemented. The falling position of the elderly was determined through GPS, and fall events could be detected based on gravitational acceleration and altitude difference. The ECG signal was used alongside GPS to increase the accuracy of FDS. The healthcare system consisted of an ECG module, a GPS module, a microprocessor embedded in a field programmable gate array (FPGA) board, and the Zigbee module. The Zigbee transmitted the fall event and localization information to the receiving Zigbee connected with the computer server. The information in the computer was then transferred to the healthcare center via Ethernet. Results showed 95\% fall detection accuracy with ECG and 80\% localization accuracy with GPS. The outdoor healthcare monitoring system consumed more power when it continuously monitored for subject fall and position. The system was suitable for outdoor applications; however, GPS would not work in indoor environments [26].

A combination of accelerometer and electromyography was considered in Reference [27] to monitor the daily activity of the elderly and patients and to detect their falls. The proposed method 
achieved 98\% recognition accuracy. Force sensor fusion and accelerometer decision were merged to efficiently to detect the fall of elderly people. The force sensor could detect, locate, and track elderly people, as well as identify their activities such as standing, sitting, walking, falling, lying down, and the transitions between their activities. Zigbee wireless protocol was employed to send the information of the force and accelerometer sensors to the neighboring node and any agent node. However, the system was costly and consumes a large amount of power because the accelerometer and force sensors are installed under tiles in large numbers. In Reference [28], a wireless patient monitoring system was proposed to monitor the patient fall, ECG signal, and indoor location of the fall incident. An accelerometer was used to monitor the patient fall, and indoor positioning was determined based on Zigbee wireless mesh networks. The proposed system consisted of sensor node, router node, and sink node. When a fall occurred, the vital signs of the patient were transmitted to the sink node through the Zigbee network. The Zigbee was configured to enter sleep mode in normal conditions to save energy and woke up during an abnormal event to transmit the patient's vital signs. With the sleep/wake strategy, the Zigbee could work continuously for $30 \mathrm{~h}$ using an $800 \mathrm{mAh}$ battery.

Fall detection was distinguished from the daily activities of the subject in Reference [29] by using six artificial intelligence techniques, namely, support vector machine (SVM), k-nearest neighbor (k-NN), Bayesian decision making, least squares method (LSM), ANN, and dynamic time warping. Six sensor units were used to detect falls. Each unit supported a single intelligent technique and included three sensors (i.e., accelerometer, magnetometer/compass, and gyroscope). Sensor measurements were transmitted to the remote PC through Zigbee wireless protocol. The authors deduced that the k-NN and LSM achieved the best results of $99 \%$ in terms of accuracy, specificity, and sensitivity. In Reference [30], a sensor node that could precisely detect human movement and fall was developed to meet size, weight, and responsiveness requirements by employing data from gyroscopes, accelerometers, and magnetometers. Zigbee wireless protocol was used to create a WBAN and then transmit the collected data from body sensors to a remote server (RS) when the subject was inside the home. The access points inside the home were created through Zigbee to reduce the power consumption of the network. Conversely, when the subject is outside his or her home, the data could be transmitted from WBAN to RS via Bluetooth using a smartphone device.

In Reference [31], a fall detection wearable device was proposed based on an accelerometer sensor, FPGA, and Zigbee wireless protocol. A low-cost algorithm was implemented in FPGA to detect the fall event. Zigbee sends raw data about the fall to an outside computing board for data analysis every $100 \mathrm{~ms}$. The power consumption of the wearable device is minimized with the sleep/wake scheme, where Zigbee is activated only when a fall event happens. Otherwise, it is deactivated to conserve the energy of the wearable device. The power consumption of Zigbee was approximately 190 and $9 \mathrm{~mW}$ in active and deactivate modes, respectively. In Reference [32], a wearable elderly fall detection prototype was designed and presented. A novel algorithm was implemented inside a microcontroller to detect the fall of a subject. A wearable system relay on the sensor node includes a three-axis accelerometer, a microcontroller, and the Zigbee wireless protocol. Subject fall detection data could be transmitted to the gateway (i.e., portable computer) via Zigbee. Experimental results indicate that the system had $96.25 \%$ accuracy, sensitivity of $98.75 \%$, and specificity of $94.38 \%$. However, the power consumption of the sensor node increased once the sampling rate was set to $100 \mathrm{~Hz}$.

Recently, the authors of Reference [33] studied the best location of fall detection sensors (i.e., gyroscope, accelerometer, and magnetometer) on a patients' body to ensure a minimum number of sensor nodes in the proposed system, and employed Zigbee for data transmission. The authors concluded that the waist is the best location for a single fall detection sensor, achieving $99.96 \%$ sensitivity based on the k-NN algorithm. In Reference [34], a homecare system was designed and implemented for fall detection and localization in a hospital. A tri-axial accelerometer was employed for fall detection, and the fall algorithm was embedded in a microcontroller for analyzing the accelerometer measurements. The proposed homecare system wakes up every second (taking advantage of the 
internal interruption of the microcontroller) to transmit the fall and location information to nursing staff through the IEEE 802.15.4 standard.

The k-NN algorithm was presented in Reference [35] to develop a smartphone device for fall detection and warning. The proposed system consists of a smartphone and a wearable motion sensor platform integrated with a gyroscope, a three-axis accelerometer, a microcontroller, and a Bluetooth module. The proposed system was tied to a custom T-shirt worn by the elderly people to measure the angular velocity and acceleration of their daily living activities in real time. The velocity and acceleration data were sent via Bluetooth to a smartphone, which engages a program relay on the kNN algorithm to examine data and sense falls in the background. The smartphone then sent messages to the health center via the GSM network. A high fall detection accuracy of $97.7 \%$ was obtained, and specificity and sensitivity are $99 \%$ and $94 \%$, respectively. In Reference [36], the physiological parameters (i.e., pulse rate, blood pressure, and temperature) of elderly people were monitored in addition to their falls. Falls were detected by a wrist sensor device, which included an accelerometer, a microcontroller, and a Bluetooth module. Physiological and fall information are sent to a tablet $\mathrm{PC}$ via Bluetooth to inform the medical staff about the conditions of the elderly people. Irregular conditions were automatically recognized, and warning messages were transmitted to the health center in real time. Experimental results demonstrated that the fall detection accuracy was $80 \%$. Sensitivity and specificity were $87.5 \%$ and $75 \%$, respectively, for a hard fall from standing position to the floor. However, the wrist sensor device was expected to consume a considerable amount of power because it continuously monitors physiological parameters and elderly falls during the test period.

An FDS based on Bluetooth module, microcontroller (ATmega88, Atmel, San Jose, CA, USA), and accelerometer (ADXL345B, Analog Devices, MA, USA) was designed and implemented by Oporto and Vilcahuaman [37]. The Bluetooth module sent an alert signal about the fall event through a mobile telephone that then transmitted an SMS to a health center or ambulance to request medical assistance. Low-power components were selected to reduce the overall power consumption of the FDS. A gyroscope and a three-axis accelerometer were integrated into a wearable sensor system (WSS) in Reference [38] to detect the fall of elderly adults. The WSS includes sensor modules (i.e., gyroscope and three-axis accelerometer), a microcontroller unit module, and a Wi-Fi module. The WSS was located on the chest of the subject to gather real-time motion information on daily activities such as walking, stepping, running, and falling. A detection algorithm was developed to precisely identify the activities of the elderly. The WSS transmitted the subject fall information to the receiving computer for real-time analysis of angular velocity and acceleration. Experimental results indicated that the proposed algorithm detects the subject's fall relative to other daily activities with a sensitivity of $96.3 \%$ and a specificity of $96.2 \%$. However, the power consumption of the module (i.e., Wifly RN131) was still high and consumed $40 \mathrm{~mA}$ in receiving mode and $210 \mathrm{~mA}$ in transmitting mode [39].

Currently, smartphone devices that comprise a wide set of implanted components include several sensors such as accelerometers, digital compasses, microphones, cameras, GPS, and gyroscope components. Wearable equipment and the acceleration measurement for fall detection can be supported because of the gradual decrease in smartphone cost. Accordingly, several smartphone devices have been used for fall detection over the last few years, such as in References [40-48]. However, in Reference [40], extra power was consumed by the Android monitoring system, as indicated by battery drain analysis in an assessment of the feasibility of FDSs [40]. The location of the subject was estimated based on tag and cellular localization [49] inside a hospital because localization accuracy is less critical in homecare systems. The objective of localization is to determine the presence of the subject in a particular area inside the hospital. Results indicated that fall sensitivity and specificity were $98.33 \%$ and $97.77 \%$, respectively. The homecare monitoring system functioned for 3 weeks or approximately $490 \mathrm{~h}$ and could constantly monitor two elderly persons round the clock.

In Reference [50], a novel fall detection algorithm was developed to monitor movements and to distinguish a fall from regular daily activities of patients using a wearable device. In case the patient falls, the wearable device automatically transmits the patient's location and requests caregivers for 
assistance. The software and hardware investigation of the wearable device essentially relied on a single accelerometer, microcontroller, GPS, and GSM module. An efficient fall detection quaternion algorithm was designed for outdoor applications with minimal resources. The wearable device was designed to work for 2 days only. Other approaches used to detect fall incidents are the use of a camera and SVM [51-53]. In Reference [51], the proposed FDS differs from others because it can work even in dark conditions. SVM was employed to determine if fall motion occurs. On the basis of the SVM method, the proposed method outperformed state-of-the-art methods in terms of accuracy $(97.6 \%)$, with sensitivity and specificity of $95.3 \%$ and $100 \%$, respectively.

Some studies such as References [54-56] adopted multiple-input multiple-output array [50] and Doppler radar [51,52] to sense and identify elderly fall and no-fall situations. However, the radar method has the following limitations and challenges: (i) it responds to any movement from other humans or non-human sources; (ii) the radar is exposed to jitter, which generates time-dependent additional noise and false person tracking; (iii) the detected person must be within the position of the antenna beam width; (iv) the reflected signal caused by the target is blocked by furniture; and (v) the person detection distance might represent a restriction, where the reflected signal is weak when the distance is increased [56]. A novel approach was presented in Reference [57]. It used fuzzy logic to identify the range and type of elderly fall, which includes position before fall, fall direction, fall velocity, and post-fall inactivity. Fuzzy logic facilitates elderly fall detection and categorizes it into one of the potential fall patterns. In addition, fuzzy logic could reduce the restrictions in parameter evaluation to introduce flexible and smooth decisions. Experimental results show that the proposed approach could sense dynamic and beacon falls and distinguish actual falls from wrongly identified falls.

The work presented here differs from these previous studies because it combines the measurements of tilt sensor, accelerometer sensor, and shock sensor to decide whether a fall event is happening or not. If the fall is real, then the FDS sends an alert signal to the $\mathrm{CN}$ to ensure that proper medical care is delivered instantly. The elderly fall incident is accurately estimated based on established artificial intelligence techniques such as ANN. The ANN was considered because of its minimum localization error as well as for its efficiency and speed compared to others localization techniques such as fingerprinting techniques. Where the fingerprinting techniques require memory cost, the setup is time consuming and any variation in network configuration like the adding of a new beacon, will imply forming a new database, meaning this technique is not so flexible. The FDS includes a simple standalone microcontroller (based on ATmega328P) and Zigbee wireless protocol in addition to the above three sensors. All these elements are embedded in a single sensor node. The option of a standalone microcontroller is considered to reduce the power consumption of the proposed system. The power consumption is significantly reduced by implementing a DDA, where the Zigbee transmits the data in the case of the subject falling and sleeps the rest of the time. Moreover, the size, weight, and complexity of the proposed system are minimized.

\section{Methodology}

\subsection{Wireless Sensor Network for Elderly Fall Detection}

The proposed elderly fall and localization WSN topology is applied in two indoor environment scenarios, namely, LOS and NLOS. A test area with a size of $40 \times 30 \mathrm{~m}^{2}$ is the location for the trial implementation of the system, as shown in Figure 1. The WSN consists of six nodes; three beacon nodes (N1, N2, and N3), a sensor node (i.e., FDS), a router node (RN), and CN or sink node. Each beacon node includes Zigbee wireless protocols (XBee Series 2) powered from the main source, eliminating concerns about power consumption. The beacon nodes N1, N2, and N3 are fixed at the coordinates $(0,0),(0,30)$, and $(40,15)$, respectively, to allow the collection of different RSSI values for ANN training, testing, and validation as shown in Figure 1. The beacon nodes are fixed on a surface $1.5 \mathrm{~m}$ above the ground to avoid the effect of the boundary of the first Fresnel Zone, $h_{O}\left(h_{O}=0.5 \sqrt{\lambda d}\right)$. Where, in the current work, $\lambda$ is the wavelength of the transmitted signal of the adopted $2.4 \mathrm{GHz}$ wireless protocol 
and $d$ is the distance between the antennae of the beacons and the sensor node (carried by the subject). The ground-reflected signal is available in the first Fresnel zone when $h_{O}$ is greater than or equal to the antenna height. The ground-reflected path has a significant effect on the power of the received signal [58]. For $2.45 \mathrm{GHz}, h_{O}$ is $0.125 \mathrm{~m}$ at a distance of $0.5 \mathrm{~m}$ between beacons and the subject, $0.958 \mathrm{~m}$ at a distance of $30 \mathrm{~m}$, and $1.24 \mathrm{~m}$ at a distance of $40 \mathrm{~m}$. Consequently, the height of the beacons nodes must be greater than $1.24 \mathrm{~m}$, and thus the signal from the beacons can arrive intact at the sensor node.

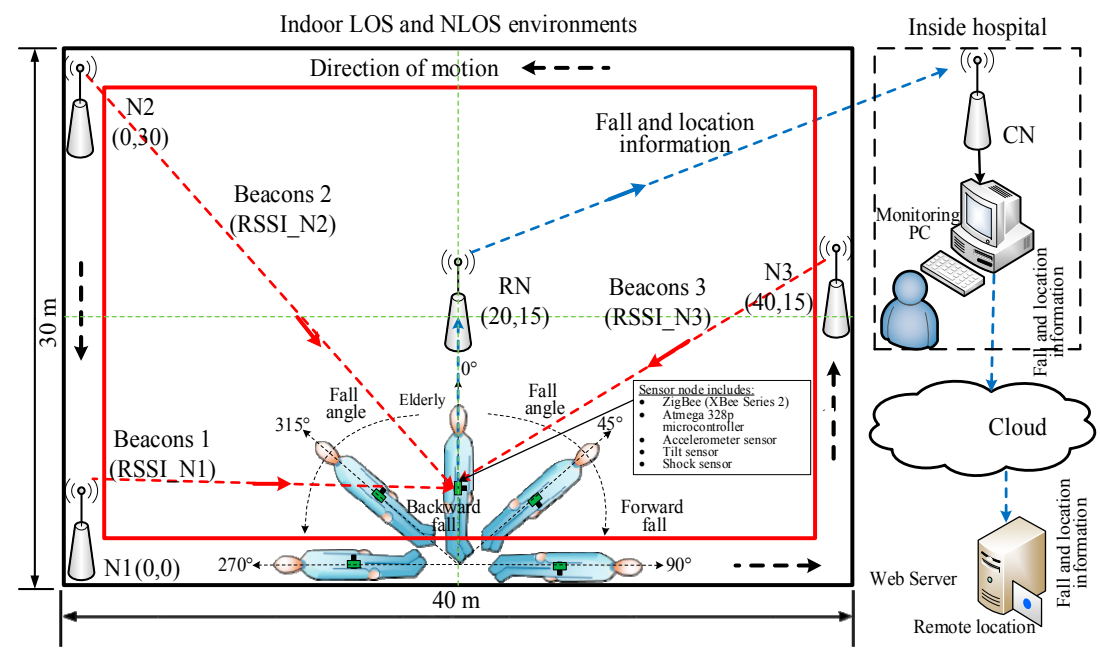

Figure 1. Topology of WSN of the subject fall and localization system.

The FDS shown in Figure 2 is wrapped around the waist of the subject, as shown in the snapshot in Figure 3a. The FDS comprises a tilt sensor (mercury switch), accelerometer sensor (ADXL 355B), shock sensor, microcontroller ATmega328P as a barebones unit to reduce the power consumption of the FDS, Zigbee wireless protocol (XBee Series 2), and a power unit (i.e., 3.7 V/1000 mAh rechargeable battery), as illustrated in Figure $3 \mathrm{~b}$. The RN is fixed on the ceiling in the middle of the hall to ensure communication with the FDS and the $\mathrm{CN}$. All the distances between nodes in the network lie within the range of the XBee Series 2, which can achieve a $100 \mathrm{~m}$ communication range between two nodes [59]. The RN passes all fall and location information of the subject to the $\mathrm{CN}$. The $\mathrm{CN}$ monitors the activities (fall and location) of the elderly person and sends the data to the caregiver during emergency cases. The $\mathrm{CN}$ can be connected to the gateway to transmit the information of the elderly person to remote locations through the Internet of Things platform, as shown in Figure 1.

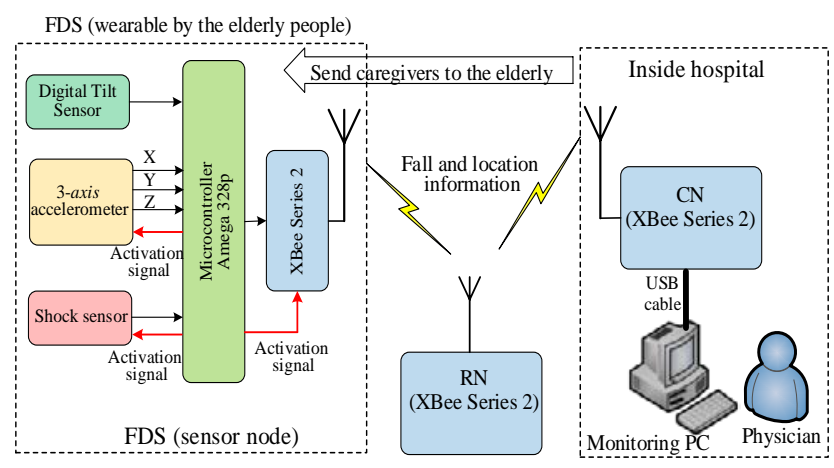

Figure 2. FDS architecture.

The FDS is shown in Figures 2 and 3, and introduces a simple hardware implementation which includes minimal interface and connection between sensors to the microcontroller and the wireless protocol. The digital tilt sensor and the shock sensor each have three connection pins for a physical interface with the microcontroller: supply voltage, ground, and output. Two pins are utilized to power 
the sensors with $+3.3 \mathrm{~V}$ and the third pin is the digital and analog serial output signal for a tilt sensor and shock sensor, respectively. However, the accelerometer sensor requires two extra pins compared to the tilt and shock sensors. The microcontroller of the sensor node of the FDS is connected with the XBee S2 module via two pins, one for the data bus and the other for activating the XBee S2. The design of the router node is very simple, needing only to pass data from the sensor node to the coordinator node through the pin for the receive data $(\mathrm{Rx})$ to the pin of the transmit data $(\mathrm{Tx})$ using one wire. The XBee S2 of the coordinator node is connected to the PC though an Arduino microcontroller using the USB cable. It is clear that the structure of the FDS is uncomplicated.

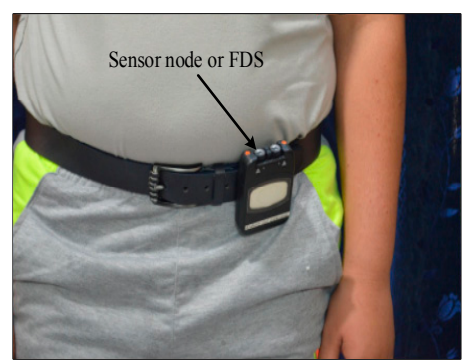

(a)

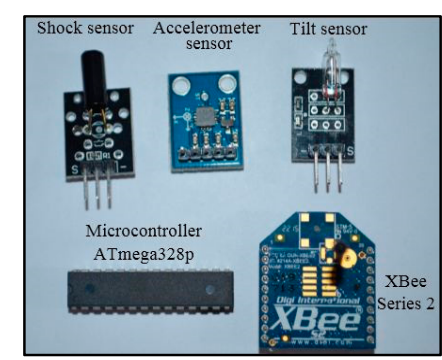

(b)

Figure 3. Proposed FDS (a) fastened to the subject individual's waist, (b) its components.

\subsection{Indoor Environment}

The simulation was performed in the test area, as shown in Figure $4 a, b$. The two figures show the WSN topology explained in the previous section. The elderly subject carried the FDS and moved in a rectangular path inside the test area. Two scenarios, namely, LOS and NLOS, were considered in this study. The LOS scenario is shown in Figure 4a. The test area was proposed to be empty to ensure direct barrier-free communication between nodes in the network. The same area and subject path of LOS was adopted in the NLOS scenario illustrated in Figure $4 \mathrm{~b}$ for a fair comparison of localization error. However, many obstacles or barriers such as walls, windows, and doors were proposed in the NLOS environment, as shown in Figure $4 \mathrm{~b}$. The FDS collected the fall information and raw data of the RSSI from three beacon nodes and transmitted them to the $\mathrm{CN}$ via $\mathrm{RN}$. Consequently, the $\mathrm{CN}$ supported by a monitoring system such as PC, tablet, and notepad can estimate the location of the fallen subject to be sent to the caregivers.

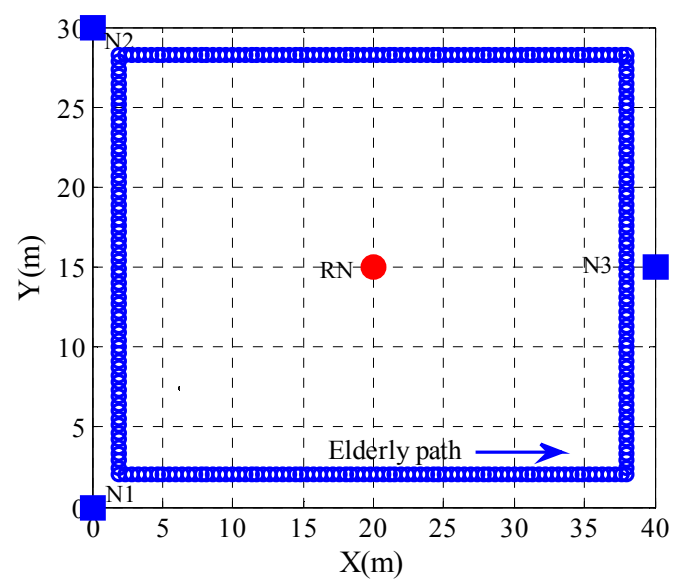

(a)

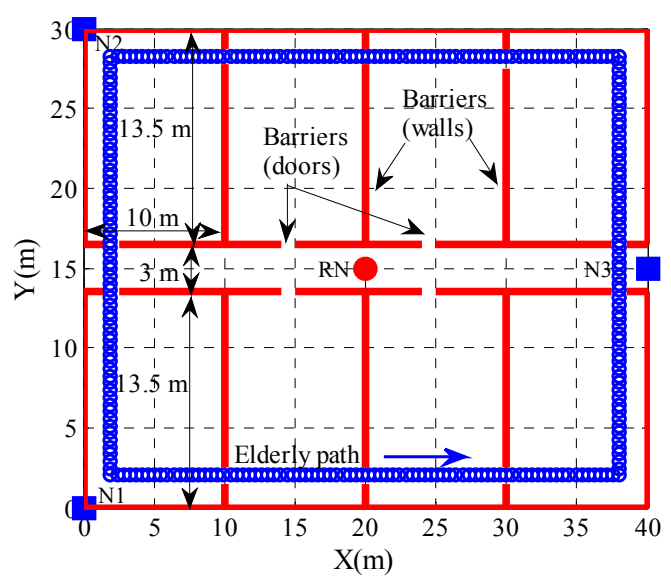

(b)

Figure 4. Adopted indoor environments (a) LOS and (b) NLOS. 


\subsection{RSSI Generation}

RSSI was generated based on the log-normal shadowing model (LNSM). LNSM was employed because it is a traditional wireless path loss model [60]. Other studies have considered this technique for channel modeling in outdoor and indoor surroundings to estimate the distance between the nodes in WSN [61]. Other studies considered this model for channel modeling in outdoor and indoor surroundings to estimate the distance between the nodes in WSN [61]. On the basis of the path loss model in Equation (1), the path loss of the received signals from beacon nodes N1, N2, and N3 toward the sensor node (i.e., FDS) was obtained as shown in Figure $5 a-c$, respectively, for LOS and NLOS. The figures show that the path loss increases with the distance between the beacon nodes and the sensor node. The path loss of the signal in NLOS environments is greater than that in LOS environments because the barriers present in NLOS are absent in LOS.

$$
W_{l}=W_{o}+10 \alpha \log _{10}\left(l / l_{0}\right)+\gamma \quad \text { in } \mathrm{dB}
$$

where the $W_{l}$ is the path loss power at a distance of 1 meter, $W_{o}$ is the path loss power at reference distance $l_{o}(1 \mathrm{~m}$ is recommended in most studies such as References $[21,62,63])$, and $\gamma$ is the Gaussian random variable in decibels with zero-mean and standard deviation. The basic notations used in the current paper are summarized in Table 1.

Similarly, the RSSI can be generated based on the LNSM (Equations (2) and (3))

$$
\begin{gathered}
R S S I=W_{T}-W_{l} \\
R S S I=W_{T}-W_{o}-10 \alpha \log _{10}\left(l / l_{0}\right)-\gamma \quad \text { in dBm }
\end{gathered}
$$

where $W_{T}$ is the transmitted power of the beacon node, and $2 \mathrm{dBm}$ is adopted for XBee Series 2.

A higher transmitted power was selected to ensure communication between the beacon nodes and the sensor node (i.e., FDS) in the adopted configuration of WSN. The RSSI raw data were collected through the moving path of the subject inside the test area. A total of 280 RSSI samples from 280 locations between each beacon node and the sensor node were recorded. A total of 840 RSSI samples were collected to train, test, and validate the ANN for subject localization without any pre-processing for the raw data. Figure $6 \mathrm{a}-\mathrm{c}$ show the RSSI with respect to the distance between the sensor node and beacon nodes N1, N2, and N3, respectively. The NLOS environment clearly affected the path loss of the wireless link more than the LOS environment did. Figures 5 and 6 show convergence between the LOS and NLOS plots at close distances. However, the divergence between these plots increased with the distance because several barriers or obstacles in the path of the signal caused signal degradation.

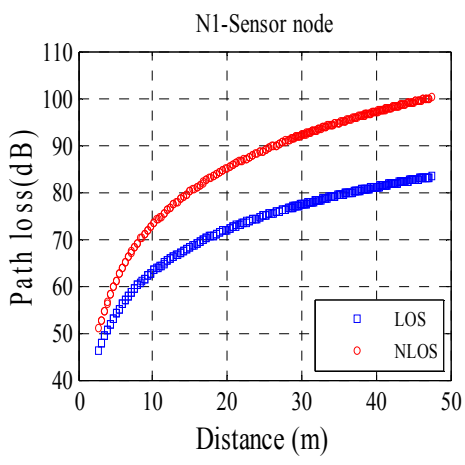

(a)

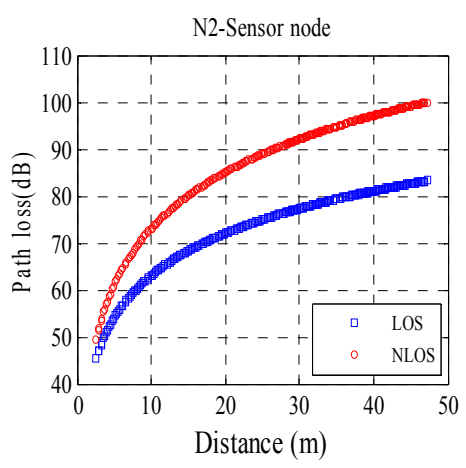

(b)

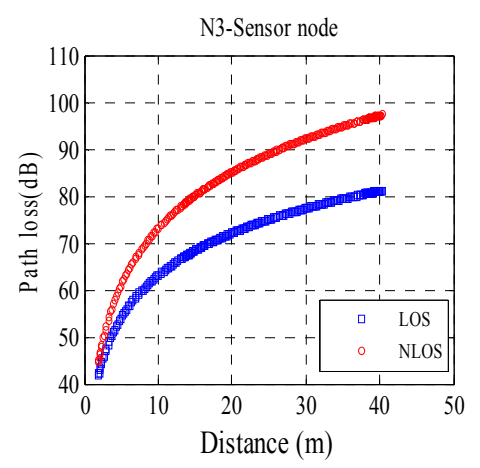

(c)

Figure 5. Path loss between beacon nodes and sensor node for LOS and NLOS (a) N1-sensor node, (b) N2-sensor node, and (c) N3-sensor node. 
Table 1. Notations and definitions.

\begin{tabular}{|c|c|c|c|}
\hline Symbols & Definitions & Symbols & Definitions \\
\hline$W_{l}$ & Path loss power & $I_{A v g \_T L}$ & Average current consumption of the tilt sensor \\
\hline$W_{o}$ & Path loss power at reference distance $=1 \mathrm{~m}$ & $I_{A v g_{-} A C C}$ & Average current consumption of the accelerometer \\
\hline$W_{T}$ & Transmitted power of the beacon node & $\boldsymbol{I}_{\text {Avg_SH }}$ & Average current consumption of the shock sensor \\
\hline$\gamma$ & Standard deviation & $I_{A v g_{Z} Z B}$ & Average current consumption of the ZigBee \\
\hline$l$ & Distance under test & $D C$ & Duty cycle \\
\hline$l_{o}$ & Reference distance ( $1 \mathrm{~m}$ in this study) & $I_{\text {active_TL }}$ & Active current consumption of the tilt sensor \\
\hline$x_{\text {estimated }}$ & Estimated location for the $x$ coordinate & $I_{\text {sleep_ACC }}$ & Sleep current consumption of the accelerometer \\
\hline$y_{\text {actual }}$ & Actual location for the $y$ coordinate & $I_{\text {active_SH }}$ & Active current consumption of the shock sensor \\
\hline$y_{\text {estimated }}$ & Estimated location for the $y$ coordinate & $I_{\text {sleep_SH }}$ & Sleep current consumption of the shock sensor \\
\hline$M A E_{x}$ & Mean absolute error for the $x$ coordinate & $I_{\text {active_MR }}$ & Active current consumption of the microcontroller \\
\hline$M A E_{y}$ & Mean absolute error for the $y$ coordinate & $I_{\text {sleep_MR }}$ & Sleep current consumption of the microcontroller \\
\hline$a_{y}(t)$ & Accelerations along the $y$-axis & $t_{s w}$ & Time transient from sleep to active modes \\
\hline$a_{z}(t)$ & Accelerations along the $z$-axis & $l$ & data packet length of ZigBee \\
\hline$I_{\text {avg }}$ & Average current consumption of FDS & $S$ & ZigBee data rate \\
\hline
\end{tabular}

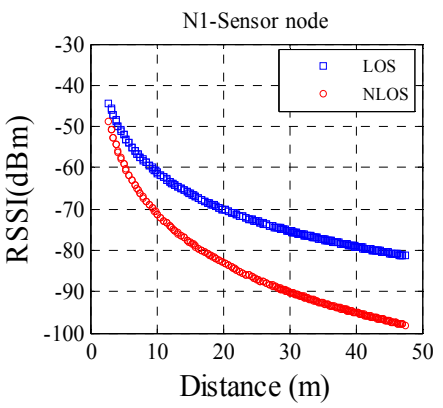

(a)

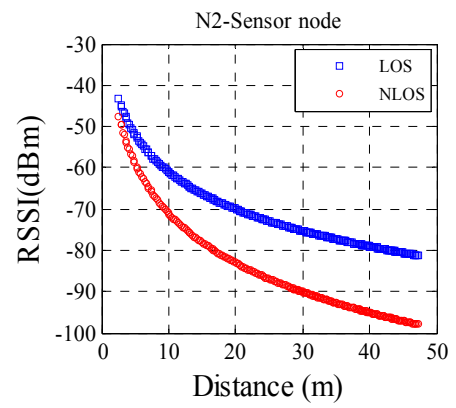

(b)

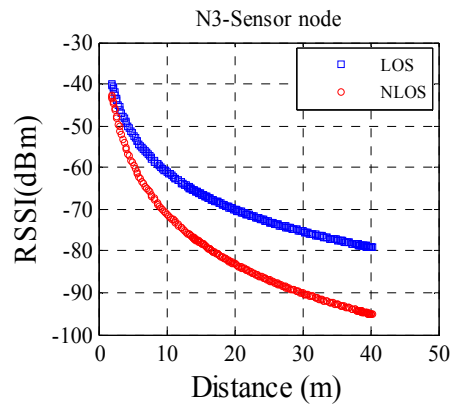

(c)

Figure 6. RSSI between beacon nodes and sensor node for LOS and NLOS: (a) N1-sensor node, (b) N2-sensor node, and (c) N3-sensor node.

\subsection{Localization Technique}

Localization techniques based on ANN can develop a complex mathematical correlation between the input and output variables. The input variables in the elderly fall detection application are the RSSI data, and the output or target variables are the elderly fall incident location $x$ and $y$. ANN was used to automatically learn nonlinear approximation algorithms. Therefore, ANN is suitable for the current application where the collected RSSIs are nonlinear data. In the current work, a backpropagation (BP) neural network-based Levenberg-Marquardt (LM) algorithm was adopted for training, testing, and validating the collected data of RSSI and the elderly fall incident location. The BP-based LM was selected because it provides the smallest localization error, as confirmed in References $[26,63,64]$, efficiency, and high convergence speed. However, it needs a large operating memory [65]. In training, testing, and validation of ANN, 840 samples of RSSI raw data were used as follows: 70\% (588 samples) for training, 15\% (126 samples) for testing, and 15\% (126 samples) for validation as recommended in References $[26,66,67]$ to accurately determine the elderly fall and incident location for LOS and NLOS environments. Before training, testing, and validating ANN data, the numbers of inputs, outputs, hidden layers with its neurons, and learning rate must be determined. In this paper, three inputs (RSSI_N1, RSSI_N2, and RSSI_N3) and two outputs ( $x$ and $y$ ) were employed.

Several hidden layers and neurons were executed to allow the ANN to provide less time consumption and minimum fitness function (i.e., mean squared error (MSE)). Fitness function and time consumption were compromised to obtain the best number of hidden layers and neurons in 
ANN. The ANN was trained, tested, and validated to obtain the MSE performance for 5, 10, 15, and 20 neurons in each hidden layer for LOS and NLOS as shown in Figures 7 and 8, respectively. Several neurons in each hidden layer are implemented to allow the ANN to choose the neurons that can perform the minimum MSE and elapsed time. Figures $7 \mathrm{~d}$ and $8 \mathrm{~d}$ show that 20 neurons provide the best performance for the ANN because they achieve a MSE of approximately $9.447 \times 10^{-6}$ (validation) and $3.447 \times 10^{-3}$ (validation) after 100 epochs for LOS and NLOS, respectively. With regards to the other neurons, 5, 10, and 15 neurons demonstrate high MSE. On the other hand, one hidden layer was trained, tested, and validated for 5, 10, 15, and 20 neurons to investigate the performance of ANN. Figure $9 \mathrm{a}, \mathrm{b}$ show the performance of MSE of validation data for the LOS and NLOS using one hidden layer, respectively. The figures exhibit lower MSE performance than two hidden layers, where they achieve a MSE of 0.00195 and 0.1177 at 100 epochs for LOS and NLOS, respectively. As a result, two hidden layers, each of which includes 20 neurons, are considered in this study because it achieves lower MSE. The learning rate (LR) (i.e., $L R=0.535$ ) was selected to achieve the minimum fitness function for indoor environments as proven in Reference [26]. One-hundred iterations were chosen to reduce the time consumption of ANN. According to these parameters, the ANN architecture, shown in Figure 10, was adopted to determine the elderly fall location. The mean absolute error (MAE) was utilized as a fitness function of the ANN algorithm to evaluate the localization error in $x$ and $y$ locations as illustrated in Equations (4)-(6).

$$
\begin{gathered}
M A E_{x}=\frac{1}{n} \sum_{i=1}^{n}\left|x_{\text {actual }}-x_{\text {estimated }}\right| \\
M A E_{y}=\frac{1}{n} \sum_{i=1}^{n}\left|y_{\text {actual }}-y_{\text {estimated }}\right| \\
M A E=\frac{M A E_{x}+M A E_{y}}{2}
\end{gathered}
$$

where $n$ is the number of tested samples (i.e., 280 samples for each input), $x_{\text {actual }}$ and $y_{\text {actual }}$ are the actual locations for the $x$ and $y$ coordinates, and $x_{\text {estimated }}$ and $y_{\text {estimated }}$ are the estimated locations for $x$ and $y$ coordinates obtained from ANN. The details of the ANN process based on the previous description and equations are presented in the flow diagram in Figure 11.

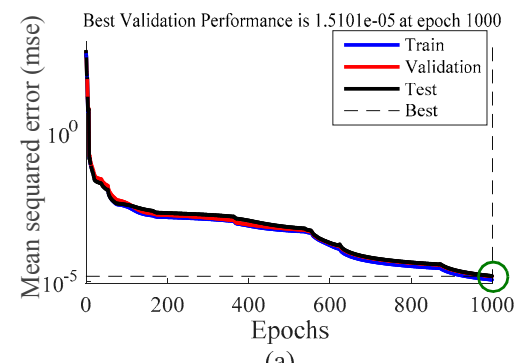

(a)

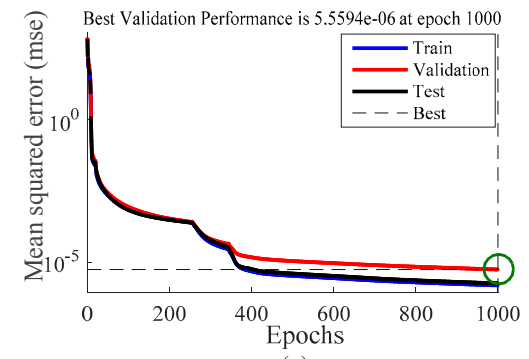

(c)

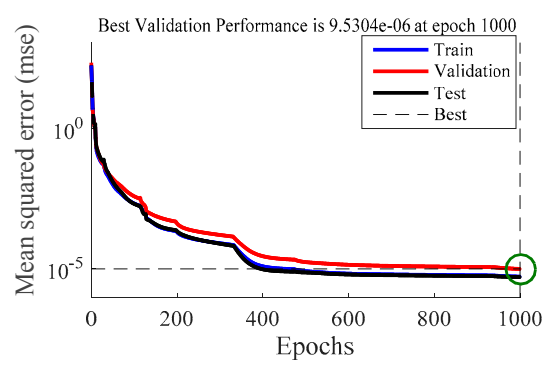

(b)

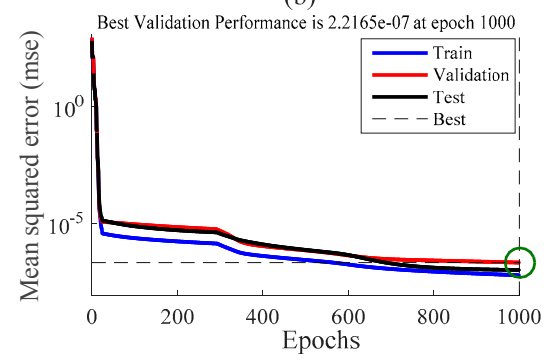

(d)

Figure 7. Performance of MSE of training, testing, and validation data for the LOS environments for (a) 5, (b) 10, (c) 15, and (d) 20 neurons. 


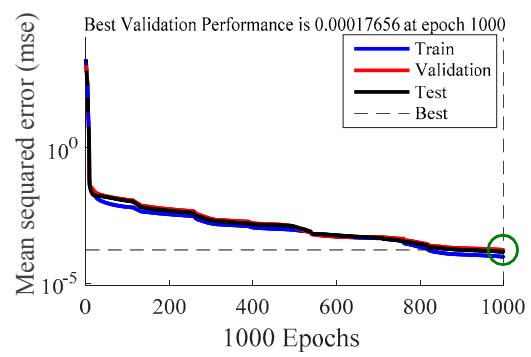

(a)

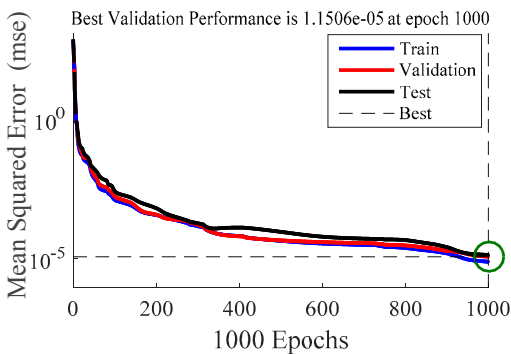

(c)

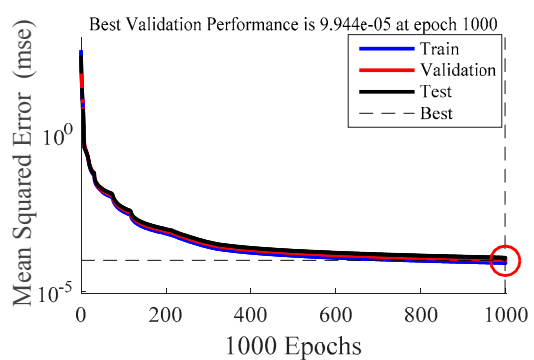

(b)

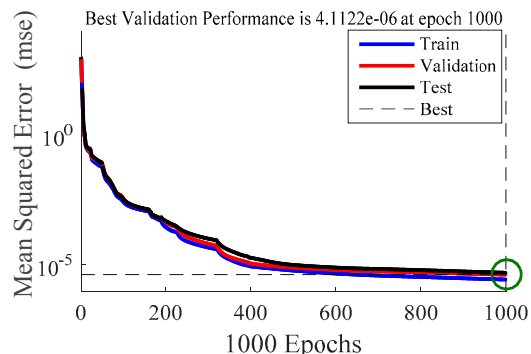

(d)

Figure 8. Performance of MSE of training, testing, and validation data for the NLOS environments for (a) 5, (b) 10, (c) 15, and (d) 20 neurons.

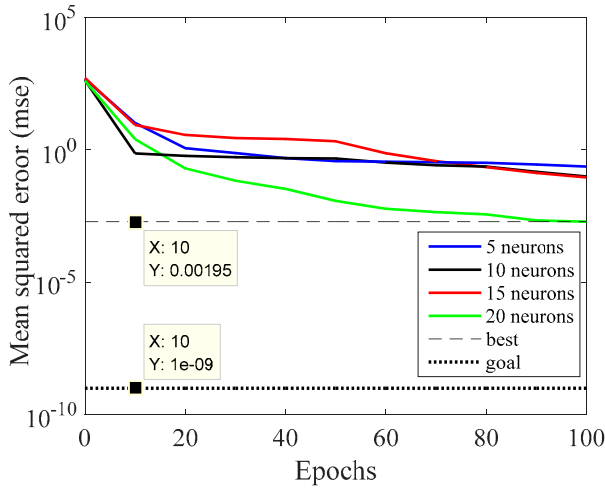

(a)

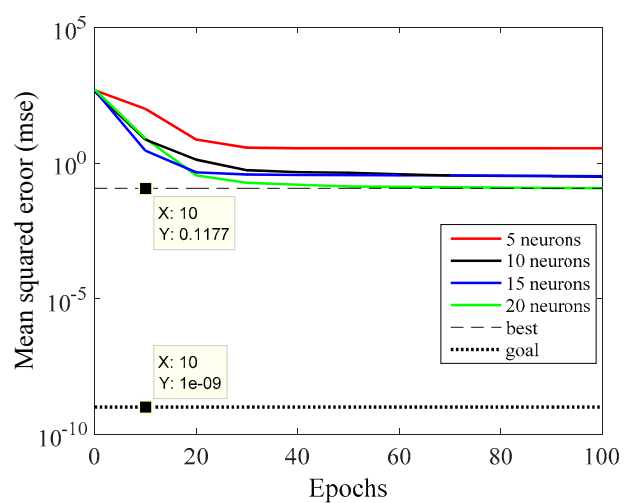

(b)

Figure 9. Performance of MSE of validation data for the (a) LOS and (b) NLOS environments using one hidden layer.

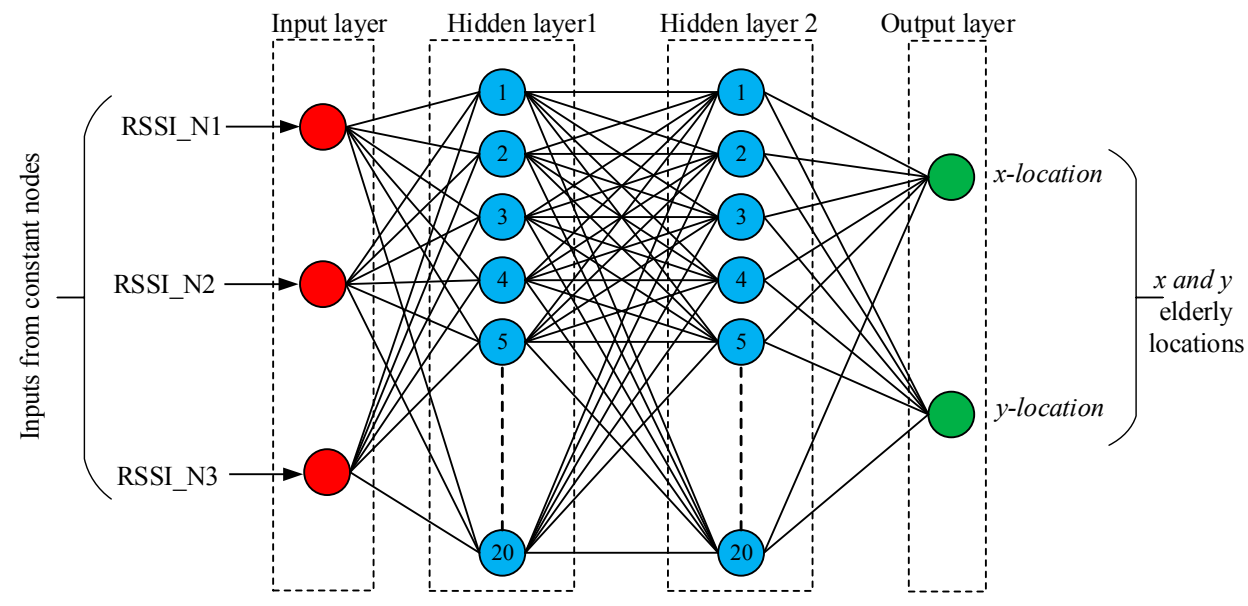

Figure 10. Architecture of adopted ANN. 


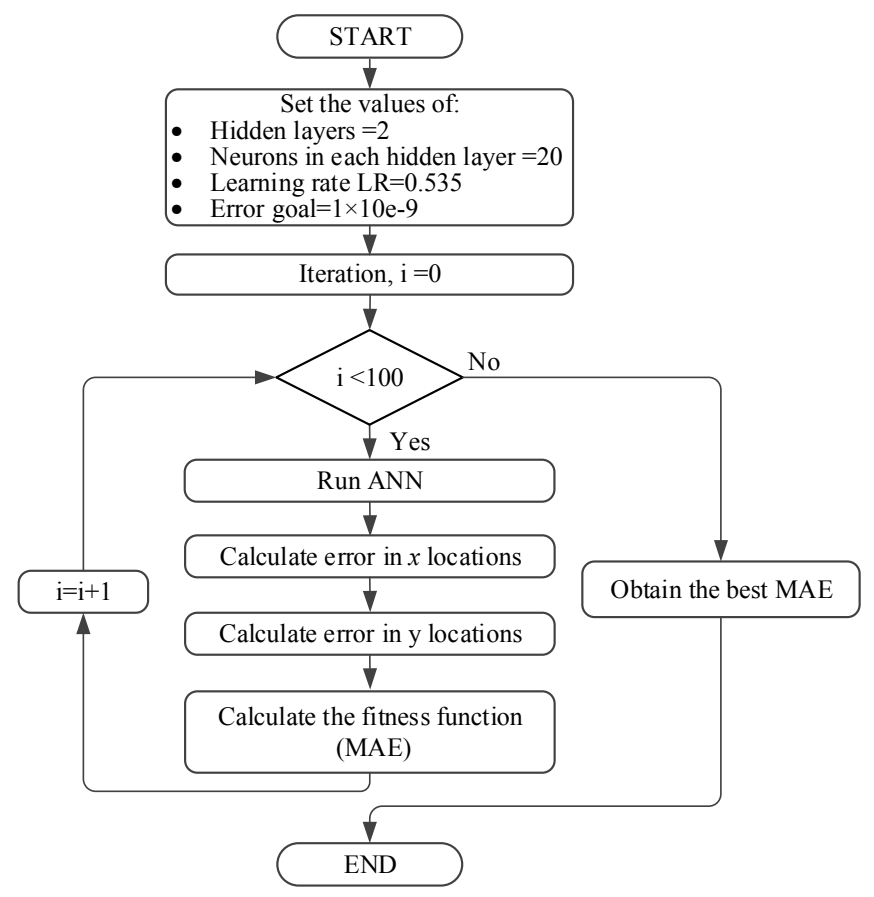

Figure 11. Flow diagram of ANN.

\subsection{Fall Detection and Data-Driven Algorithm}

S-BFDA and DDA are merged into one algorithm (Algorithm 1) and launched inside the microcontroller to reduce the power consumption and computational process of the sensor node microcontroller. Algorithm 1 shows the S-BFDA as dashed-line boxes and DDA as dash-dot boxes. The S-BFDA is based on the threshold of the (i) tilt sensor, (ii) angle of incidence, and (iii) shock to the ground when their peak values are above or below the threshold. The tilt sensor sends an interrupt digital signal (Logic 1) to activate the microcontroller once the elderly fall happens in one of the following situations: (i) backward fall, (ii) forward fall, (iii) right-side fall, and (v) left-side fall. The microcontroller in turn activates the accelerometer and shock sensors by supplying them with power and sends a digital control signal to awaken the Zigbee. On the basis of Cartesian coordinates $x y z$ for the subject shown in Figure 12, the accelerometer sensor can be used to measure the angle of fall $\theta(t)$ of the subject by applying Equation $(7)[68,69]$.

$$
\theta(t)=\cos ^{-1}\left(\frac{a_{x}(t)}{\sqrt{a_{x}(t)^{2}+a_{y}(t)^{2}+a_{z}(t)^{2}}}\right)
$$

where $a_{x}(t), a_{y}(t)$, and $a_{z}(t)$ are the accelerations along the $x, y$, and $z$ axes, respectively.

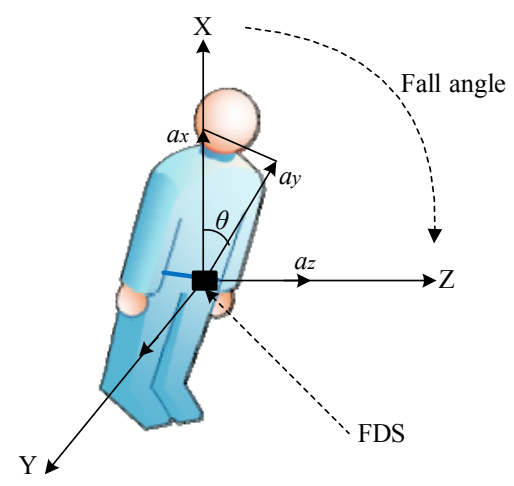

Figure 12. Geodetic coordinates of the elderly. 
The shock sensor detects the elderly shock to the ground, and the tilt sensor detects the inclination of the subject during the fall. However, real-time fall detection may be compromised by false-alarm data from lying and falling positions. The S-BFDA is proposed to overcome this problem by fusing the tilt, angle, and shock measurements. Accordingly, the fall detection accuracy is satisfactory, and results indicate that the proposed FDS is energy efficient and can be used for accurate fall detection. In the proposed DDA, the components of the sensor node (i.e., sensors and Zigbee) are deactivated except for the microcontroller, which operates in low-power mode. The microcontroller continues to work in low-power mode to monitor the elderly fall on the basis of the tilt sensor. If the angle (which is derived from the accelerometer reading Equation (7)), shock, and tilt values exceed their threshold levels, an alert signal is sent to the $\mathrm{CN}$ via $\mathrm{RN}$. In other words, upon a change in logic from ZERO to ONE for tilt and shock sensors and an angle of more than $45^{\circ}$, the microcontroller activates the Zigbee wireless protocol to transmit the fall event. The Zigbee simultaneously measures the RSSI values of the three beacon nodes. Once the measurements are completed, the FDS transmits an alert signal, including the fall of the elderly and the collected RSSI values, to the CN via the RN. When the transmission process is complete, the FDS returns to sleep mode to save energy. The strategy of switching between wake and sleep modes will significantly improve the power consumption of the sensor node. The sensor node transmits data infrequently and briefly and sleeps most of the time.

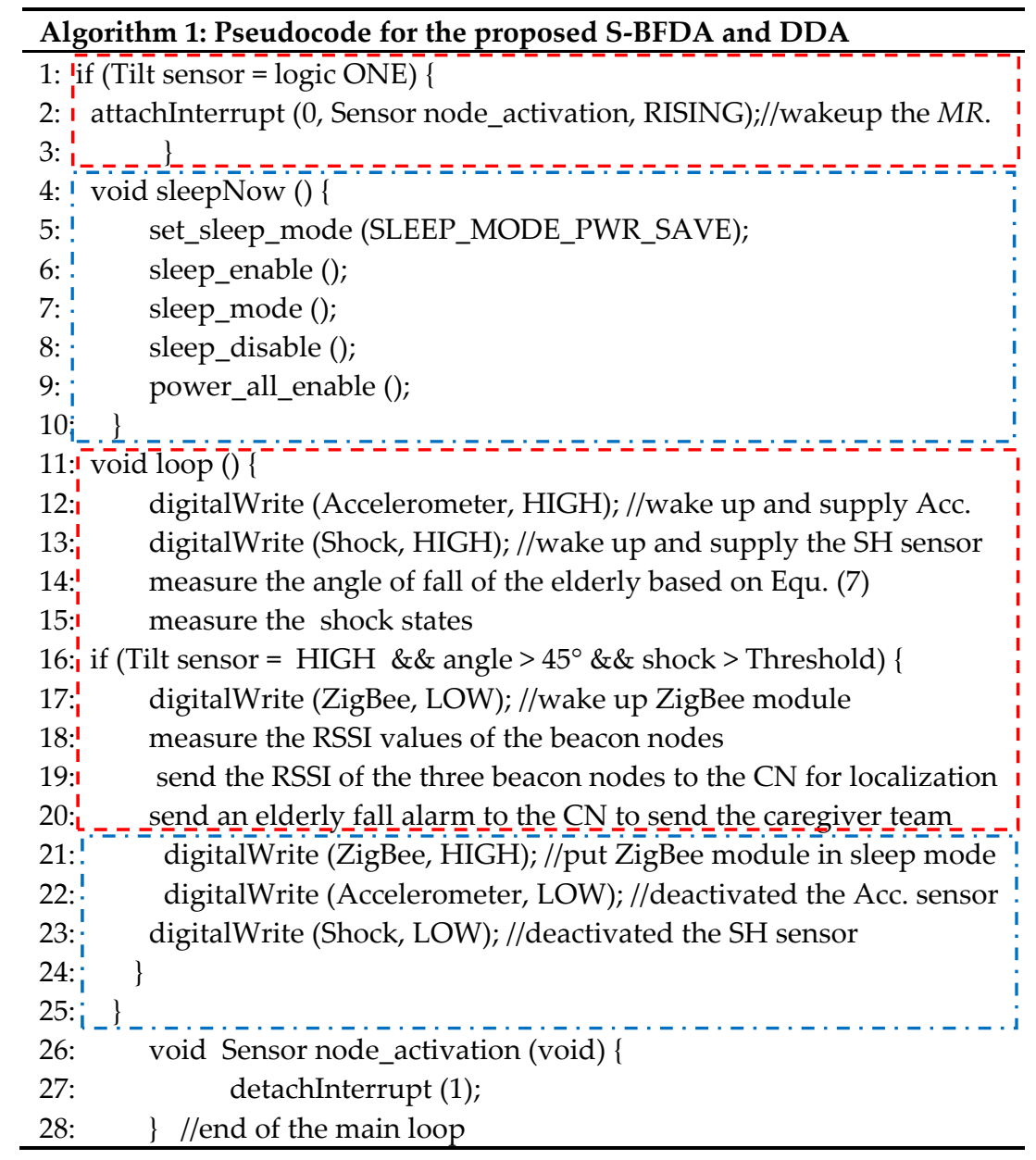

\section{Power Consumption Analysis}

The current and time consumption for each component of the FDS is measured by using an ampere meter for sleep and active modes, as shown in Table 2. The table illustrates the current 
consumption for each component of the FDS. Subsequently, the average current consumption $\left(I_{\text {avg }}\right)$ of the FDS of $676 \mu \mathrm{A}$ is acquired by applying Equations (8)-(10) [70].

$$
I_{A v g}=I_{A v g_{T L}}+I_{A v g_{A C C}}+I_{A v g_{S H}}+I_{A v g_{M R}}+I_{A v g_{Z B}}
$$

where $I_{A v g_{-} T L}, I_{\text {Avg_ACC }}, I_{\text {Avg_SH }}, I_{\text {Avg_MR }}$, and $I_{\text {Avg_ZB }}$ are the average current consumption of the tilt sensor, accelerometer sensor, shock sensor, microcontroller, and Zigbee model, respectively. The average current consumption of each element of the FDS can be expressed as follows:

$$
\left.\begin{array}{c}
I_{\text {Avg_TL }}=D C \times I_{\text {active_TL }}+(1-D C) I_{\text {Sleep_TL }} \\
I_{\text {Avg_ACC }}=D C \times I_{\text {active_ACC }}+(1-D C) I_{\text {sleep_ACC }} \\
I_{\text {Avg_SH }}=D C \times I_{\text {active_SH }}+(1-D C) I_{\text {sleep_SH }} \\
I_{\text {Avg_MR }}=D C \times I_{\text {active_MR }}+(1-D C) I_{\text {sleep_MR }} \\
I_{\text {Avg_ZB }}=D C \times I_{\text {active_ZB }}+(1-D C) I_{\text {sleep_ZB }}
\end{array}\right\}
$$

where $I_{\text {active }}$ and $I_{\text {sleep }}$ are the active and sleep current consumption of the element of the FDS, respectively. DC is the duty cycle, which can be calculated from the ratio of the active time $\left(t_{\text {active }}\right)$ to the total time $\left(T_{\text {total }}\right)$. The active time is at least $1 \mathrm{~min}$ to cover the fall detection event, and the total time is $24 \mathrm{~h}(1440 \mathrm{~min})$ assuming the subject falls once a day.

Table 2. Current and time consumption of each component of the FDS.

\begin{tabular}{cccccc}
\hline Parameter & Tilt Sensor & Accelerometer Sensor & Shock Sensor & Microcontroller @ 4 MHz & ZigBee (XBee S2) \\
\hline$I_{\text {active }}(\mathrm{mA})$ & 0.4 & 0.27 & 0.5 & 2.5 & 37.5 \\
$I_{\text {sleep }}(\mathrm{mA})$ & 0 & 0 & 0 & 0.09 & 0.56 \\
$t_{\text {active }}(\mathrm{min})$ & 1 & 1 & 1 & 1 & 1 \\
${ }^{*} t_{\text {sleep }}(\mathrm{min})$ & 1439 & 1439 & 1439 & 1439 & 1440 \\
$T_{\text {total }}(\mathrm{min})$ & 1440 & 1440 & $6.4 \times 10^{-4}$ & $6.4 \times 10^{-4}$ & 1440 \\
$\mathrm{DC}$ & $6.4 \times 10^{-4}$ & $0.4 \times 10^{-4}$ & 0.322 & 91.553 & $5.4 \times 10^{-4}$ \\
$I_{\text {subtotal }}(\mu \mathrm{A})$ & 0.258 & 0.174 & & \\
Average current consumption based DDA $=676 \mu \mathrm{A}$ & & \\
Total current consumption in conventional operations $=41.17 \mathrm{~mA}$ & \\
${ }^{*} t_{\text {sleep }}=T_{\text {total }}-t_{\text {active, }}$ all components working at +3.3 Volts. \\
\hline \multicolumn{7}{l}{}
\end{tabular}

The sleep current of the tilt, accelerometer, and shock sensors is equal to zero because these sensors are supplied with power from the microcontroller, as shown in Algorithm 1. Accelerometer and shock sensors are supplied with power only when the elderly fall event happens. Thus, Equation (8) will become

$$
\begin{aligned}
I_{\text {Avg }}=D C \times I_{\text {active_TL }}+D C \times I_{\text {active_ACC }}+D C \times I_{\text {active_SH }} & \\
& +\left[D C \times I_{\text {active_MR }}+(1-D C) I_{\text {sleep_MR }}\right]+\left[D C \times I_{\text {active_ZB }}\right. \\
& \left.+(1-D C) I_{\text {sleep_ZB }}\right]
\end{aligned}
$$

The total current consumption of the sensor node in conventional operation (i.e., without any power reduction algorithm) is approximately $41.17 \mathrm{~mA}$. The total current consumption of the FDS in typical operation is significantly higher than the average current consumption of the FDS when the DDA is applied. Therefore, this algorithm appears to be promising for such applications as it extends the battery life and saves a large amount of energy. Equation (11) [71] can be used to compute the percentage of power savings of the FDS relying on DDA. As a result, the performance of the FDS in terms of power consumption is proven.

$$
\begin{aligned}
\text { Power savings }(\%) & \\
= & \left(1-\frac{\text { Current consumption based on DDA }}{\text { Current consumption based on conventional operation }}\right) \\
\times & 100 \%
\end{aligned}
$$




\section{Results and Discussions}

\subsection{Fall Detection Results}

Multiple fall positions have been adopted to evaluate the performance of the S-BFDA, as shown in Figure 13. First, the data from the accelerometer, shock, and tilt sensors was collected in the standing position. The sensor data was then recorded during backward/forward, right-side, and left-side falls. We found no data from the sensors in the first case (Figure 13a). By contrast, fall information was generated by the sensors in other fall cases, as shown in Figure 13b-d. The fall detection information (i.e., inclination angle) and the RSSI values of the three beacon nodes were transmitted to the CN using the Zigbee wireless protocol via router node to be viewed on a PC. The fall detection information can be plotted in real time using the MakerPlot data acquisition software [72], as shown in Figure 14. The figure shows that $7 \mathrm{~s}$ are required from standing to the fall case.

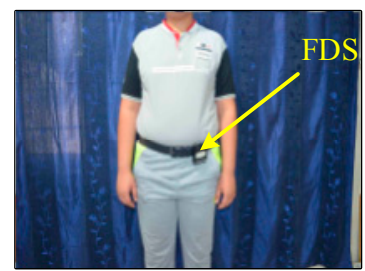

(a)

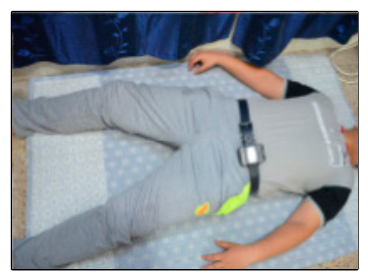

(b)

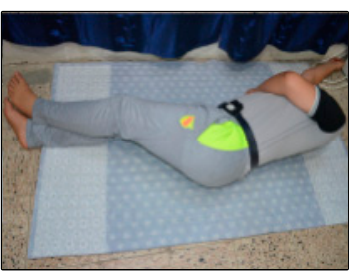

(c)

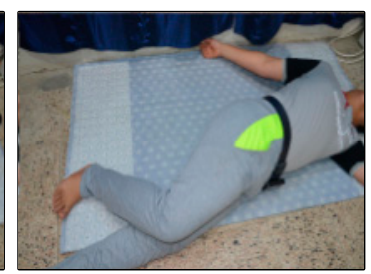

(d)

Figure 13. Different postures: (a) standing, (b) backward fall, (c) right-side fall, and (d) left-side fall.

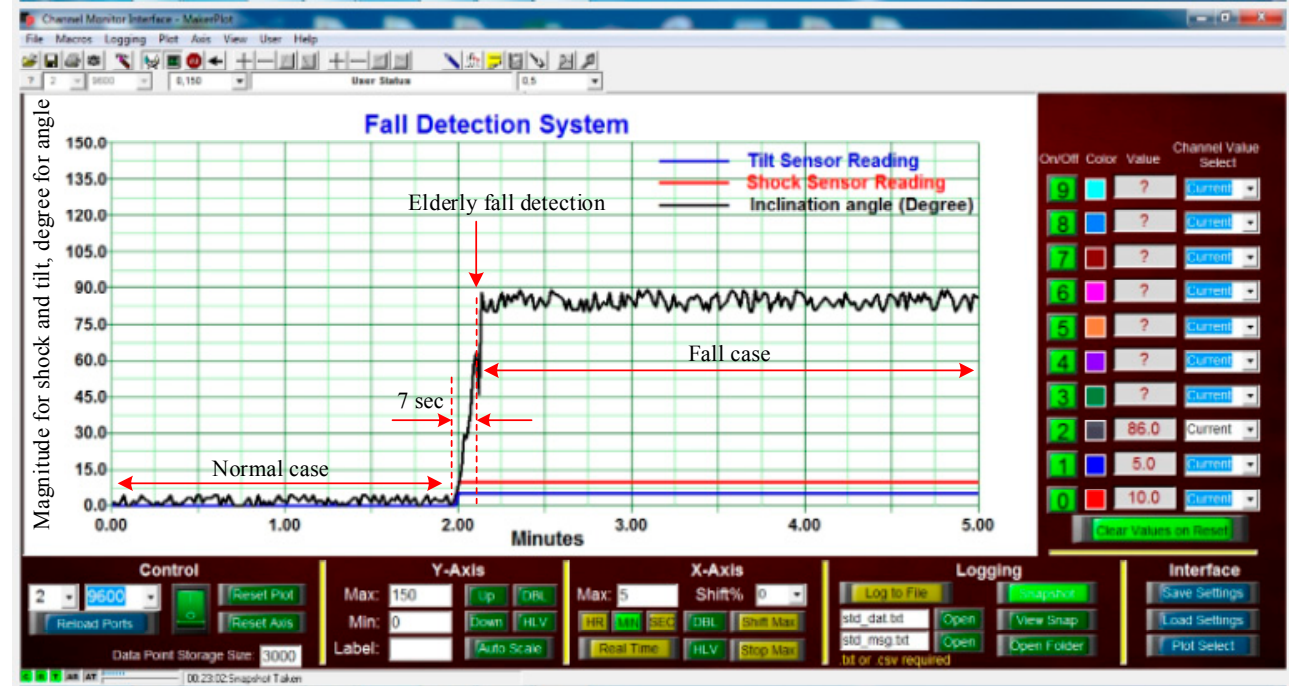

Figure 14. Plot of tilt, shock, and angle measurements based on MakerPlot software.

The data packet structure of the ZigBee consists of 127 bytes maximum, 31 bytes (overhead) and 96 (payload) [70]. In our application, the transmitted data packet length of FDS includes 42 bytes. The payload consists of 11 bytes and 31 out of 42 bytes for overhead. The payload bytes, namely, (i) four bytes for identification of the beacon nodes and angle of fall, (ii) two bytes for RSSI1 value of the N1 node, (iii) two bytes for RSSI2 value of the N2 node, (iv) two bytes for RSSI3 value of the N3 node, and (iv) two bytes for angle of fall. The data rate of the ZigBee is $250 \mathrm{Kbps}$, and therefore the transmission time $\left(t_{x}\right)$ of XBee S2 can be calculated based on Equation (11). Consequently, the active transmission time for ZigBee in the sensor node of the FDS is $11.554 \mathrm{~ms}$. Given that, the time 
from the standing case to the fall case is $7 \mathrm{~s}$ as shown in Figure 11, therefore the total time required for transmitting alarm information and localization is $7.11554 \mathrm{~s}$.

$$
t_{x}=t_{S w}+\frac{l}{S}
$$

where $t_{S W}$ is the transient time of ZigBee from sleep mode to active mode. XBee S2 consumes $10.2 \mathrm{~ms}$ when hibernate pin sleep is used. $l$ is the data packet length (i.e., $42=336$ bits) of XBee S2 in bits, and $S$ is the XBee S2 data rate.

The experiment was conducted in LOS and NLOS because daily activities occur in both environments. Such a procedure was adopted to observe the effects of these surroundings and evaluate the accuracy of the proposed system. Therefore, the accuracy of the elderly fall location is $100 \%$ (all 40 fall events were detected for LOS) and 92.5\% (3 out of 40 fall events were deviant for NLOS) as illustrated in Table 3. These results are expected because of path loss increases, and the received power attenuates with distances more in NLOS than in LOS, as seen in Figures 5 and 6.

Table 3. Fall detection accuracy of the proposed sensor-based approach.

\begin{tabular}{ccccc}
\hline Environments & Fall Detected (FD) & Fall Not Detected (FND) & $\begin{array}{c}\text { False Alarm (\%) (False } \\
\text { Alarm = FDN/FD) }\end{array}$ & $\begin{array}{c}\text { Accuracy (\%) } \\
\text { (Accuracy = FD/TE) }\end{array}$ \\
\hline LOS & 40 & 0 & 0 & 100 \\
NLOS & 37 & 3 & 8.1 & 92.5 \\
\hline
\end{tabular}

TE: Total event $=40$.

\subsection{Elderly Localization Results}

Two scenarios were implemented to investigate the localization of the subject on the basis of Zigbee WSN using a MATLAB simulation. The first scenario was conducted in the LOS environment, and the other scenario was applied in the NLOS environment.

\subsubsection{LOS Environment}

The performance of ANN can be evaluated based on the regression line and MSE. Figure 15 shows the correlation between the actual locations (i.e., target, which represents pre-defined locations) on the $x$-axis and estimated locations (i.e., output obtained from ANN) on the y-axis for training, testing, validation, and all data. The regression coefficient $(\mathrm{R})$ in the figure can be adopted in evaluating the elderly fall localization accuracy of the estimated location. The figure reveals that the correlation between the actual and estimated locations agree with each other for all cases. The R shows a linear fit, and its value is 1 . Figure 16 shows the development of the MSE of the ANN during the training, testing, and validation phases. The MSEs at the final point (i.e., 100 epochs) are $2.287 \times 10^{-6}, 4.929 \times 10^{-6}$, and $2.841 \times 10^{-6}$ for training (dash), testing (dot), and validation (dash-dot), respectively. The performance of the training data is lower than the goal set (i.e., $2.841 \times 10^{-6}$ ) beyond 85 epochs, as shown in Figure 16, implying a successful end of the suggested ANN. The performance plot indicates the following: (i) a small MSE, (ii) the validating MSE is better than the testing MSE, and (iii) no overfitting during testing and validation.

Figure 17 shows the histogram errors for the ANN model of training (blue bar), testing (red bar), and validation (black bar) data. Outliers can be employed to define the quality of the specified data. In the proposed system, a large amount of collected data (i.e., 840 RSSI values) are considered for training, testing, and validation to improve the localization accuracy and neural network operation. Each input of the neural network employs 280 RSSI values. Hence, few outlier data points are observed in the histogram plot (Figure 17). More errors are distributed between -0.00391 and 0.00417 . However, the highest errors for training and testing data points are 0.00659 and 0.00901 , respectively, relative to 
the rest of the dataset errors. The minimum error of 0.000132 is concentrated at 135 bins for the three types of dataset.

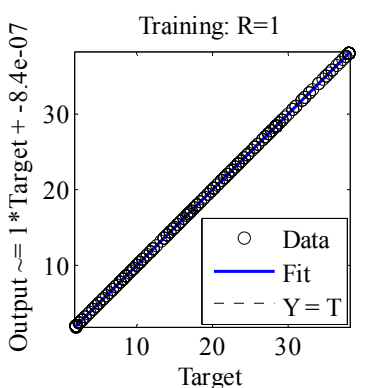

(a)

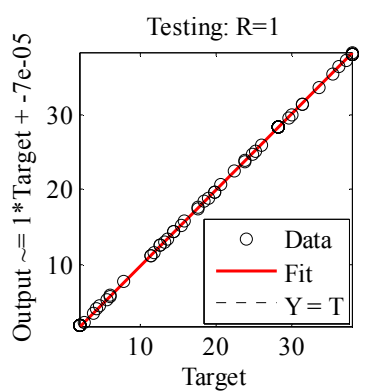

(b)

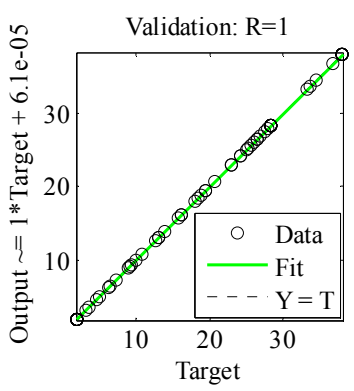

(c)

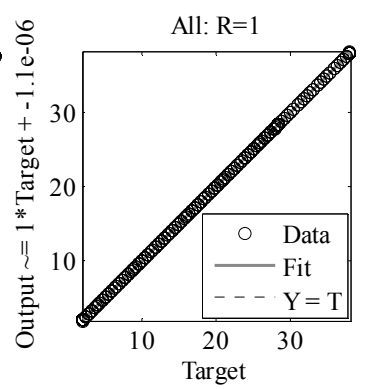

(d)

Figure 15. Regression coefficient (R) of the ANN algorithm for (a) training, (b) testing, (c) validation, and (d) all data for the LOS environments.

Best Validation Performance is $2.8414 \mathrm{e}-06$ at epoch 100

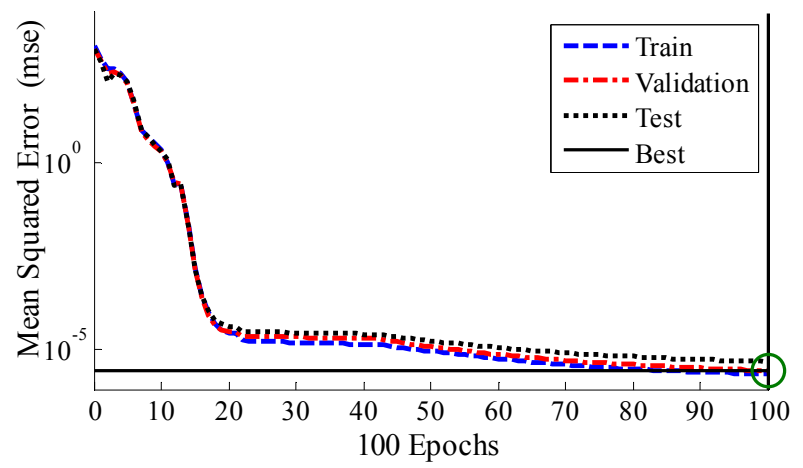

Figure 16. Performance of MSE of training, testing, and validation data for the LOS environments.

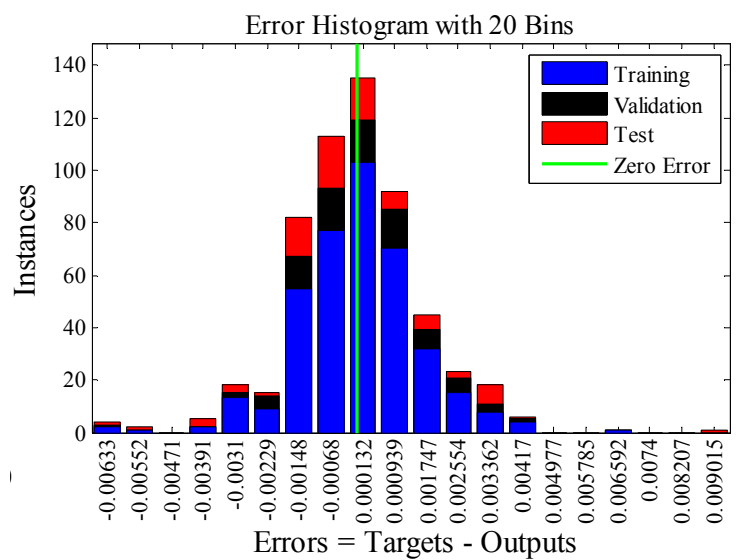

Figure 17. Histogram errors for the ANN model of training, testing, and validation data for LOS environments.

Forty-two out of 280 positions were used to estimate the elderly fall location in evaluating the subject localization based on ANN. A total of 280 RSSI values collected from each beacon node were used for training $(70 \%)$, testing $(15 \%)$, and validating $(15 \%)$ the neural network. After training, the ANN was employed for testing and validation to locate 42 unknown positions (the approximate spacing between positions is of the order of $3 \mathrm{~m}$ ) in the $30 \times 40 \mathrm{~m}^{2}$ test area, as shown in Figure 18 . The figure shows the estimated and actual locations of 42 positions represented by circle and square symbols, respectively. Minimal difference between the actual and the estimated locations was observed. 
The MAE was approximately $0.0094 \mathrm{~m}$ for LOS environments. As a result, the suggested localization technique can produce accurate elderly fall localization. Figure 19a,b show the errors in the $x$-axis and $y$-axis, respectively. Both figures show small estimated errors for $x$ and $y$ locations, as obtained from ANN.

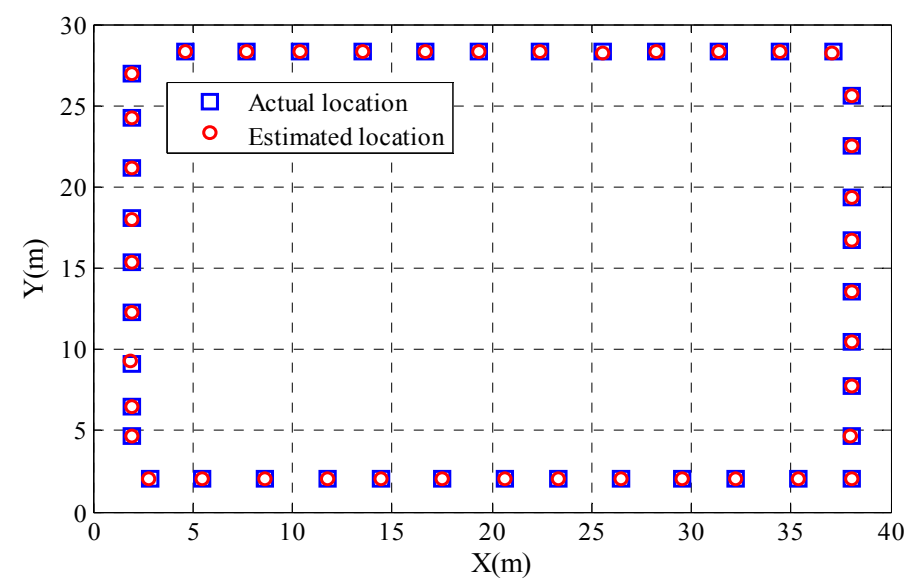

Figure 18. Actual and estimated locations for indoor LOS environments.

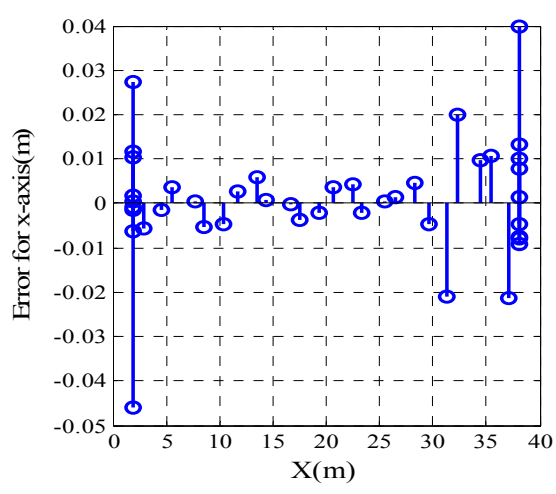

(a)

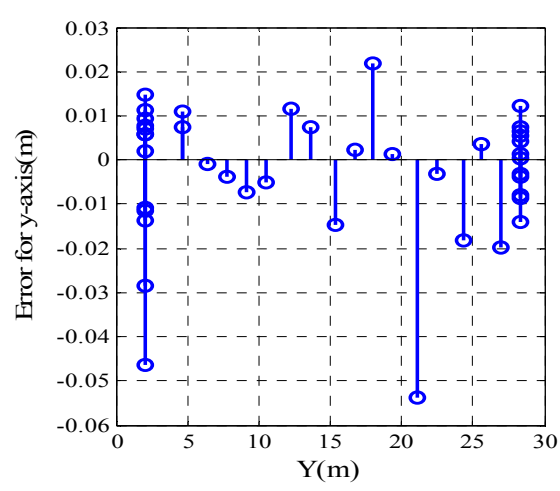

(b)

Figure 19. Elderly fall location errors at LOS environments for the (a) $x$-axis and (b) $y$-axis.

\subsubsection{NLOS Environment}

Similar to LOS environments, the same procedures can be adopted to evaluate the performance of ANN and the localization accuracy of elderly fall in NLOS environments. The performance of ANN can be estimated based on R and MSE. Figure 20 shows the correlation between the actual (i.e., target, which represents pre-defined locations) and estimated locations (i.e., output obtained from ANN) for training, testing, validation, and all data. The figure reveals that the correlation between the actual and estimated locations slightly deviates from each other, with its value being 0.997 for all cases. Figure 21 shows the MSE of ANN during the training, testing, and validation stages. The MSEs at the final point (i.e., 100 epochs) are $4 \times 10^{-3}, 5.607 \times 10^{-3}$, and $4.648 \times 10^{-3}$ for training (dash), testing (dot), and validation (dash-dot), respectively. The performance of the training data is lower than the goal set (i.e., $4.648 \times 10^{-3}$ ) beyond 90 epochs as shown in Figure 21, implying a successful end of training of the suggested ANN. The performance plot in terms of MSE indicates that MSE is small, the validation MSE is better than the testing MSE, and no overfitting occurred during the validation and testing process. Figure 22 shows the histogram errors for the ANN model of training (blue), testing (red), and validation (black) data. Outliers can be employed to define the quality of the specified data. The number of collected data of the NLOS is similar to that used in LOS environments. Hence, a small number of outlier data points are detected in the histogram plot (Figure 22). More errors are distributed between -0.1849 and 0.1888 . However, the highest error of 0.1888 was observed 
for training, testing, and validation data. The minimum error of -0.0079 is concentrated at 123 bins for the three types of datasets.

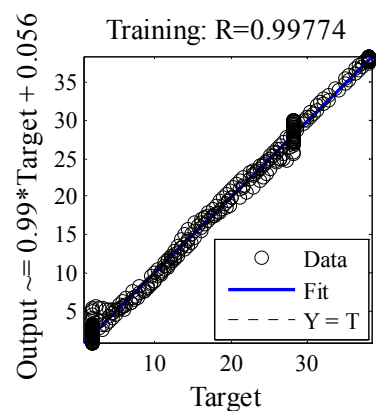

(a)

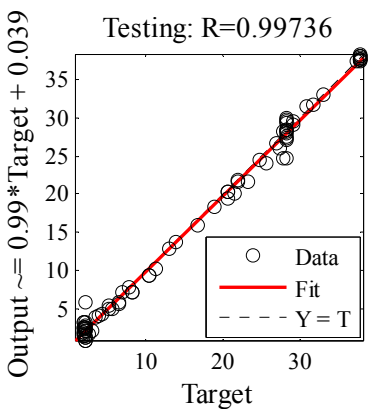

(b)

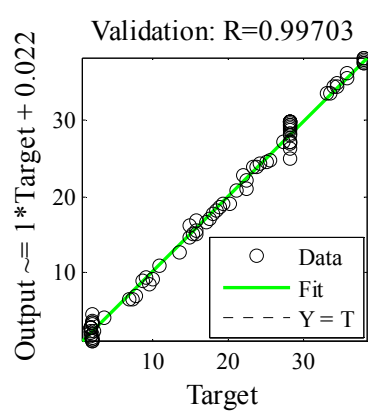

(c)

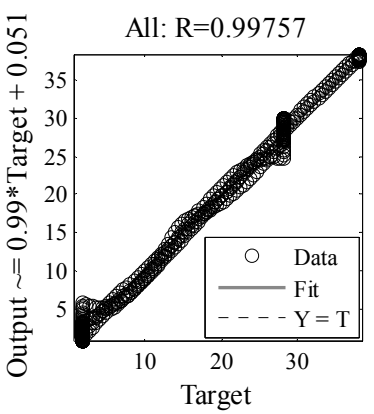

(d)

Figure 20. Regression coefficient (R) of the ANN algorithm for (a) training, (b) testing, (c) validation, and (d) all data for the NLOS environments.

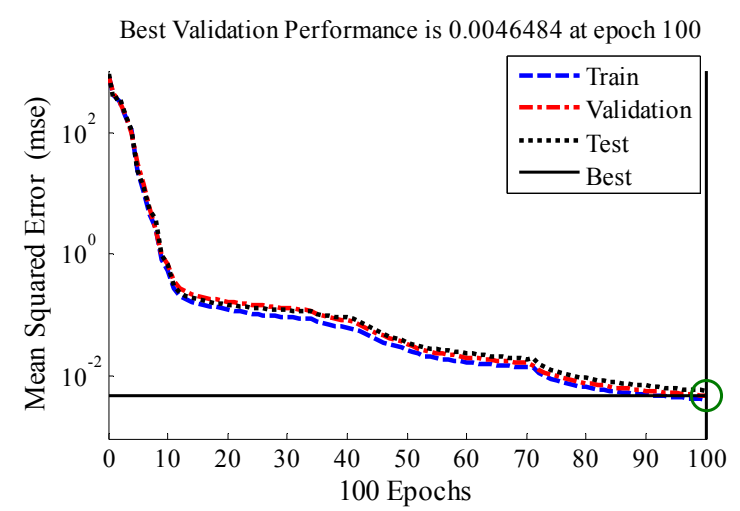

Figure 21. Performance of MSE of training, testing, and validation data for the NLOS environments.

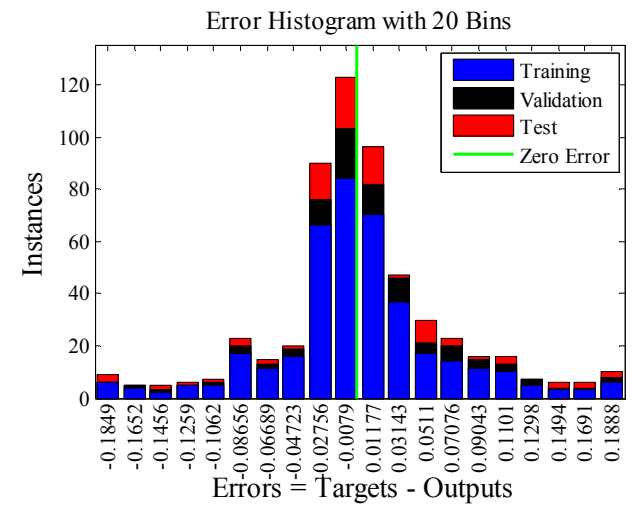

Figure 22. Histogram errors for the ANN model of training, testing, and validation data for NLOS environments.

Forty-two out of 280 positions were used to estimate the elderly fall location and evaluate the subject localization based on ANN. A total of 280 RSSI values collected from each beacon node were used for training (70\%), testing (15\%), and validating (15\%) the neural network. After training, ANN was employed for testing and validation to locate 42 unknown positions in the $30 \times 40 \mathrm{~m}^{2}$ test area, as shown in Figure 23. The figure shows the estimated and actual locations of 42 positions represented by circle and square symbols, respectively. Some circles that represent the estimated locations lie far from the actual location (square symbol). The MAE is approximately $0.0454 \mathrm{~m}$ for NLOS environments. As a result, the proposed localization technique can produce precise elderly fall localization. Figure 24a,b show the errors in the $x$-axis and $y$-axis, respectively. Both figures show 
relatively small estimated errors for $x$ and $y$ positions obtained from ANN. However, the elderly fall localization error in LOS environments is better than that in NLOS environments in terms of MAE.

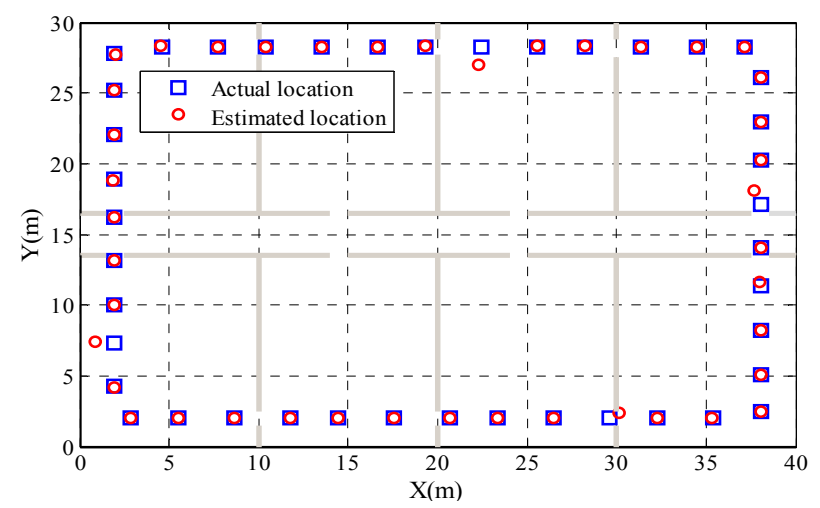

Figure 23. Actual and estimated locations for indoor NLOS environments.

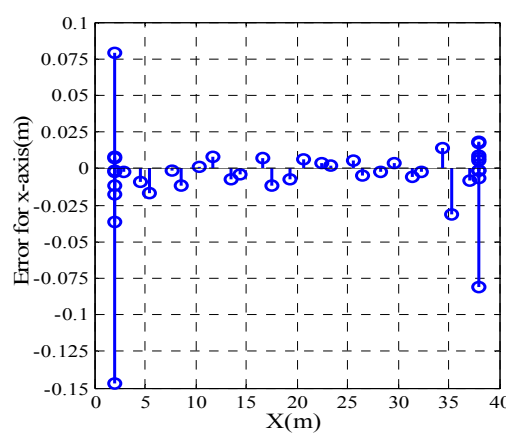

(a)

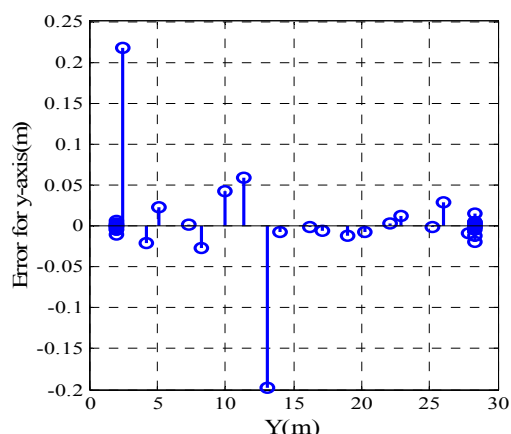

(b)

Figure 24. Elderly fall location errors at NLOS environments for (a) $x$-axis and (b) $y$-axis.

\subsection{Power Consumption Estimation Results}

DDA can reduce the power consumption of the FDS to $676 \mu \mathrm{A}$. As a result, the sensor node battery life can be prolonged to $1480 \mathrm{~h}$ (62 days) by using a lithium-ion battery with a capacity of $3.7 \mathrm{~V} / 1000$ $\mathrm{mAh}$. Consequently, power savings of $98 \%$ can be achieved for the FDS by applying Equation (11). The current consumption based on DDA is lower than that of traditional operation (without the DDA), which yields a total current consumption of $41.17 \mathrm{~mA}$. A relationship between battery capacity (on the $x$-axis) and estimated battery life (on the $y$-axis) can be established, as shown in Figure 25. The figure shows two dash-dot and dashed curves. The dashed curve represents the life of the FDS in traditional operation (without DDA), whereas the dash-dot curve represents the estimated battery life of the FDS based on DDA. The figure reveals that the power consumption of the FDS is significantly improved by using DDA.

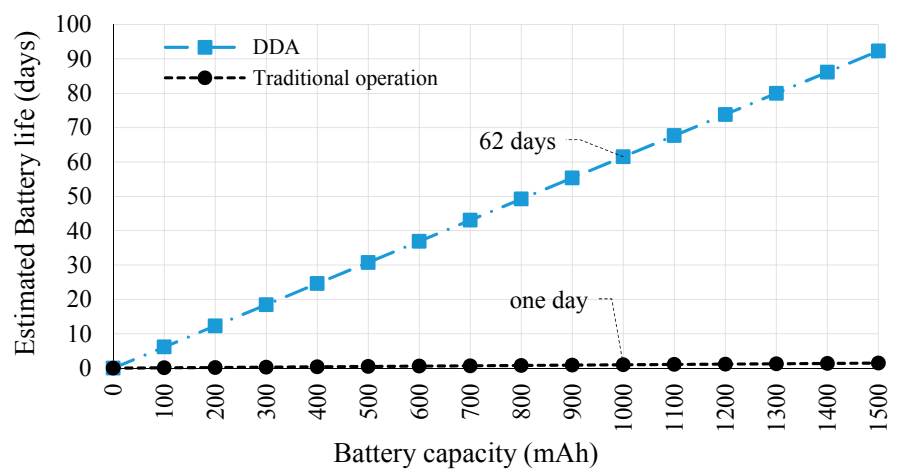

Figure 25. Battery capacity against estimated battery life for the FDS. 


\section{Comparison Results}

The comparison results in this work can be divided into three types.

\subsection{Comparison of Fall Detection Accuracy}

Fall detection accuracy in this study depends on the environment, sensor accuracy, and sensor position. When the tilt sensor in the FDS detects an elderly fall, the Zigbee is awakened and starts to measure the data of the angle of incidence, the shock that occurs when the subject falls to the ground, and the RSSI values of the three beacon nodes. The sensor node transmits an alert signal for the elderly fall and the RSSI values to the $\mathrm{CN}$ via $\mathrm{RN}$ so that a team of caregivers can be dispatched and the elderly fall can be localized. Consequently, the accuracy of the elderly fall detection in this study is comparable to that in previous studies, as shown in Table 4. The table shows different methods or algorithms that have been used for elderly fall detection along with their accuracies, adopted wireless protocols, and sensors with related positions on the body of the elderly people, as well as the test conditions. The performance of the current work in terms of fall detection accuracy is achieved based on hardware implementation (Section 3.1) and this is validated by fall detection and data-driven algorithms (Section 3.5) and measurement of fall detection accuracy on site (Section 5.1). The comparison with other related works was accomplished based on the measurement values of previous works from the literature. The performance of accuracy of previous works is presented in detail in related works (Section 2). The fall detection accuracy in this study consistently outperforms state-of-the-art methods for LOS surroundings and is similar or superior to previous works for NLOS environments, as illustrated in Table 4 . The fall detection accuracy in this study is consistent with the findings of Aziz et al. [73] and Wang et al. [74], which achieved 99.99\% and 100\% accuracy, respectively, based on SVM.

Table 4. Comparison Between the accuracy of the adopted ANN technique and fall detection algorithms in previous studies.

\begin{tabular}{|c|c|c|c|c|c|c|}
\hline Ref./Year & $\begin{array}{l}\text { Fall Detection } \\
\text { Algorithm }\end{array}$ & $\begin{array}{c}\text { Wireless } \\
\text { Protocol/Technology }\end{array}$ & Sensor Type & $\begin{array}{l}\text { Sensor } \\
\text { Location }\end{array}$ & $\begin{array}{c}\text { Test } \\
\text { Conditions }\end{array}$ & Acc. $(\%)$ \\
\hline$[47] / 2012$ & ANN & BT, IEEE 802.15 .4 & Tri-axial accelerometer & Waist & $\begin{array}{l}\text { Indoor, } \\
\text { outdoor }\end{array}$ & 81.4 \\
\hline$[75] / 2013$ & Fuzzy Logic & 3D motion analysis & Camera & $\begin{array}{l}\text { Shoulder, } \\
\text { spine, hip }\end{array}$ & N/A & 98.6 \\
\hline$[76] / 2014$ & k-NN with weighted RSSI & ZigBee & Tri-axial accelerometer & Waist & Indoor & 88.62 \\
\hline$[77] / 2014$ & Hierarchical rule-based & WiFi & $\begin{array}{l}\text { Accelerometer, GPS, } \\
\text { magnetic, orientation }\end{array}$ & Waist & Indoor & 95 \\
\hline [38]/2015 & Threshold-based & WiFi (IEEE $802.11 \mathrm{~b} / \mathrm{g}$ ) & $\begin{array}{l}\text { Tri-axial accelerometer } \\
\text { Tri-axial gyroscope }\end{array}$ & Chest & Indoor & 90 \\
\hline$[78] / 2016$ & SVM & HD camera & Camera & Head & $\begin{array}{l}\text { Indoor, } \\
\text { outdoor }\end{array}$ & 90.27 \\
\hline$[79] / 2016$ & SVM & BT & Tri-axial accelerometer & Waist & Indoor & 94.58 \\
\hline \multirow{3}{*}[80]{$/ 2016$} & Fuzzy dynamic & \multirow{3}{*}{ Kinect camera } & \multirow{3}{*}{$\begin{array}{l}\text { Accelerometer } \\
\text { Image }\end{array}$} & \multirow{3}{*}{$\begin{array}{l}\text { Camera } \\
\text { Parallel to } \\
\text { the floor }\end{array}$} & \multirow{3}{*}{ Indoor } & 92.86 \\
\hline & ANFIS and SVM & & & & & 95.71 \\
\hline & $\begin{array}{l}\text { Fuzzy beacon and } \\
\text { dynamic }\end{array}$ & & & & & 97.14 \\
\hline$[35] / 2016$ & $\begin{array}{l}\text { k-NN and sliding } \\
\text { window }\end{array}$ & BT & $\begin{array}{l}\text { Tri-axial accelerometer } \\
\text { Tri-axial gyroscope }\end{array}$ & Upper trunk & Indoor & 97.7 \\
\hline [81]/2016 & ANN, RBF, k-NN & BT & Tri-axial accelerometer & Chest & Indoor & 99 \\
\hline [82]/2017 & SVM, Threshold-based & WiFi & $\begin{array}{l}\text { Accelerometer } \\
\text { Gyroscope }\end{array}$ & $\begin{array}{l}\text { Waist, hand, } \\
\text { chest, arm }\end{array}$ & Indoor & 79.13 \\
\hline$[83] / 2017$ & $\begin{array}{l}\text { k-NN, NB, SVM, BN, DT, } \\
\text { LR, MP }\end{array}$ & Smartphone & Tri-axial accelerometer & $\mathrm{N} / \mathrm{A}$ & N/A & $81.4(\mathrm{k}-\mathrm{NN})$ \\
\hline$[84] / 2017$ & SVM, NNC, DT, DA & WiFi & $\begin{array}{l}\text { Tri-axial accelerometer, } \\
\text { Tri-axial gyroscope, } \\
\text { proximity, compass }\end{array}$ & $\begin{array}{l}\text { Left, right, } \\
\text { front-pockets }\end{array}$ & Indoor & 90 \\
\hline [85]/2017 & Compressed sensing & IEEE 802.15 .4 & Motion & N/A & Indoor & 90.9 \\
\hline
\end{tabular}


Table 4. Cont.

\begin{tabular}{|c|c|c|c|c|c|c|}
\hline Ref./Year & $\begin{array}{l}\text { Fall Detection } \\
\text { Algorithm }\end{array}$ & $\begin{array}{c}\text { Wireless } \\
\text { Protocol/Technology }\end{array}$ & Sensor Type & $\begin{array}{c}\text { Sensor } \\
\text { Location }\end{array}$ & $\begin{array}{c}\text { Test } \\
\text { Conditions }\end{array}$ & Acc. (\%) \\
\hline \multirow{3}{*}[86]{$/ 2017$} & Data-level fusion & \multirow{3}{*}{ UWB radar system } & \multirow{3}{*}{ Doppler signatures } & \multirow{3}{*}{$\begin{array}{l}\text { L-shape } \\
\text { geometry }\end{array}$} & \multirow{3}{*}{ Indoor } & 91.63 \\
\hline & Decision level fusion & & & & & 79.63 \\
\hline & Sensor selection & & & & & 86.99 \\
\hline$[87] / 2017$ & SVM & BT LE & Smartwatch & Wrist & N/A & 93.8 \\
\hline$[88] / 2017$ & SVM & Microsoft kinect & Microsoft kinect & $\begin{array}{l}\text { Skeletal } \\
\text { joints }\end{array}$ & Indoor & 93.6 \\
\hline$[89] / 2017$ & Camera-based & Microsoft lifeCam & Camera & Waist & $\begin{array}{l}\text { Indoor, } \\
\text { outdoor }\end{array}$ & 93.78 \\
\hline$[90] / 2017$ & Fall Risk Index (FRI) & Bluetooth LE & $\begin{array}{l}\text { Accelerometer, Gyroscope, } \\
\text { Pressure }\end{array}$ & Insoles & Indoor & 93.9 \\
\hline$[91] / 2017$ & Depth image & Microsoft kinect & Infrared (IR) & $\begin{array}{c}\text { Skeletal } \\
\text { joints }\end{array}$ & Indoor & 93.94 \\
\hline \multirow{2}{*}[92]{$/ 2017$} & SVM & \multirow{2}{*}{ WiFi } & \multirow{2}{*}{$\begin{array}{l}\text { Without sensors (signal } \\
\text { path) }\end{array}$} & \multirow{2}{*}{ Beacon AP } & \multirow{2}{*}{ Indoor (LOS) } & 90 \\
\hline & $\mathrm{RF}$ & & & & & 95 \\
\hline$[93] / 2017$ & SVM & Bluetooth & Accelerometer & Lumbar & $\begin{array}{l}\text { Indoor, } \\
\text { outdoor }\end{array}$ & 94.58 \\
\hline$[94] / 2017$ & k-NN & $\begin{array}{l}\text { Membership histogram } \\
\text { descriptor }\end{array}$ & Camera (video frames) & $\begin{array}{l}\text { Constant } \\
\text { camera }\end{array}$ & Indoor & 94.73 \\
\hline [95]/2017 & Vision-based & Video samples & Videos of different postures & $\begin{array}{c}\text { Constant } \\
\text { camera }\end{array}$ & Indoor & 94.9 \\
\hline$[96] / 2017$ & Ameva & Smartphone & $\begin{array}{l}\text { Electromyogram, } \\
\text { accelerometer }\end{array}$ & Hand, wrist & $\begin{array}{l}\text { Indoor, } \\
\text { outdoor }\end{array}$ & 95 \\
\hline$[97] / 2017$ & Threshold-based & $\begin{array}{c}\text { Arduino } \\
\text { UNO and Matlab }\end{array}$ & $\begin{array}{l}\text { Tri-axial accelerometer } \\
\text { Tri-axial gyroscope }\end{array}$ & Wrist & Indoor & 95 \\
\hline$[68] / 2017$ & $\mathrm{BN}$ and SW & BT class 2 & $\begin{array}{l}\text { Tri-axial accelerometer } \\
\text { Tri-axial gyroscope }\end{array}$ & Vest & $\begin{array}{l}\text { Indoor, } \\
\text { outdoor }\end{array}$ & 95.67 \\
\hline$[98] / 2017$ & Dataset & Data store in SD card & $\begin{array}{l}\text { Accelerometer and } \\
\text { gyroscope }\end{array}$ & Waist & $\begin{array}{l}\text { Indoor, } \\
\text { outdoor }\end{array}$ & 96 \\
\hline [99]/2017 & SVM & RFID & RFID Tag & Neck & Indoor & 96 \\
\hline$[100] / 2017$ & SVM, LR, k-NN, NB, DT & N/A & Tri-axial accelerometer & Waist & Indoor & 96 (SVM) \\
\hline$[23] / 2017$ & SVM, ANN & Smartphone & $\begin{array}{l}\text { Tri-axial accelerometer, } \\
\text { Tri-axial gyroscope }\end{array}$ & $\begin{array}{l}\text { Worn on the } \\
\text { body }\end{array}$ & Indoor & $\begin{array}{l}91.71 \text { (SVM) } \\
96.07 \text { (ANN) }\end{array}$ \\
\hline [101]/2017 & Hidden Markova model & WiFi (802.11 g), ZigBee & 3D image sensors & Ceiling-cameras & Indoor & 98 \\
\hline$[102] / 2017$ & VI Fusion-based, SVM & Smartphone & $\begin{array}{l}\text { Tri-axial accelerometer, } \\
\text { visual camera }\end{array}$ & $\begin{array}{l}\text { Users' } \\
\text { pocket }\end{array}$ & $\mathrm{N} / \mathrm{A}$ & 98.9 \\
\hline$[103] / 2017$ & $\begin{array}{l}\text { Threshold-based } \\
\text { Knowledge-based }\end{array}$ & N/A & Tri-axial accelerometer & $\begin{array}{l}\text { Waist, knee, } \\
\text { elbow, head }\end{array}$ & Indoor & 99.33 \\
\hline$[73] / 2017$ & SVM & $\mathrm{N} / \mathrm{A}$ & $\begin{array}{l}\text { Tri-axial accelerometer } \\
\text { Tri-axial gyroscope }\end{array}$ & $\begin{array}{l}\text { Waist, } \\
\text { sternum }\end{array}$ & Indoor & 99.99 \\
\hline$[74] / 2017$ & SVM & WiFi & $\begin{array}{l}\text { Without sensors (signal } \\
\text { path) }\end{array}$ & Beacon AP & Indoor & 100 \\
\hline $\begin{array}{l}\text { Proposed } \\
\text { S-BFDA in } \\
\text { LOS }\end{array}$ & ANN & ZigBee (XBee S2) & $\begin{array}{l}\text { Accelerometer, tilt heart } \\
\text { rate }\end{array}$ & Waist & Indoor & 100 \\
\hline $\begin{array}{l}\text { Proposed } \\
\text { S-BFDA in } \\
\text { NLOS }\end{array}$ & ANN & ZigBee (XBee S2) & $\begin{array}{l}\text { Accelerometer, tilt heart } \\
\text { rate }\end{array}$ & Waist & Indoor & 92.5 \\
\hline
\end{tabular}

Acc: Accuracy; AP: Access point; BT: Bluetooth; BN: Bayesian network; DA: Discriminant analysis; DT: Decision tree; k-NN: k-nearest neighbors; LE: Low-energy; LR: Logistic regression; MP: Multilayer perception; NB: naïve Bayes; NNC: Nearest neighbor classifiers; RBF: Radial basis function; RF: Random forest; RFID: Radio frequency identification; RSS: Received signal strength; SW: Sliding window; SVM: Support vector machine; Ultrawide band (UWB); VI: Visual and Inertial.

\subsection{Comparison of Localization Errors}

The proposed ANN localization technique can be compared with several studies that adopted different soft computing localization techniques in terms of MAE, such as particle bacterial foraging algorithm (BFA) and PSO [104], ANN [64,105-109], gravitational search algorithm hybrid with neural network (GSA-ANN) [26], PSO hybrid with neural network (PSO-ANN) [63,110], quantum swarm optimization (QPSO) [111], neuro-fuzzy (NF) and genetic fuzzy (GF) [112], and extreme learning machine (ELM) [113] for indoor environments, as shown in Figure 26. The performance of the current work in terms of MAE is achieved based on the methodology that has been presented in Section 3.2 through 3.5, and this is validated by simulation implementation (Section 3.2) and simulation results 
(Section 5.2) for LOS and NLOS environments. The comparison with other related works was achieved based on the estimated values of the previous works from the literature. The performance of the MAE of previous works is presented in Figure 26. Most of these studies are similar to the current study because they use Zigbee wireless protocol and RSSI to train data on the basis of soft computing techniques. The Zigbee wireless protocol is considered in most of these works because its RSSI can be easily measured and no extra hardware is needed. For the previous techniques, the RSSI values were used as inputs to the soft computing system, whereas the sensor node location of the coordinates ( $x$ and $y$ ) or distance between nodes were considered as outputs. The adopted elderly fall ANN localization techniques of the FDS outperform the other techniques in terms of MAE, with 0.0094 and $0.0454 \mathrm{~m}$ for LOS and NLOS, respectively, as shown in Figure 26. In this paper, a minimum localization error was obtained based on simulation in Matlab. However, in a real environment, the localization error is expected to be higher than the simulation results. These results are better than the requirements for the elderly localization system reported by Wang et al. [114], where the localization accuracy ranged between 0.5 and $1 \mathrm{~m}$ in terms of MAE. In addition, the root mean-squared error (RMSE) in the current study (0.014 for LOS and 0.0402 for NLOS) is significantly superior to that in Kianoush et al. (0.3) [101].

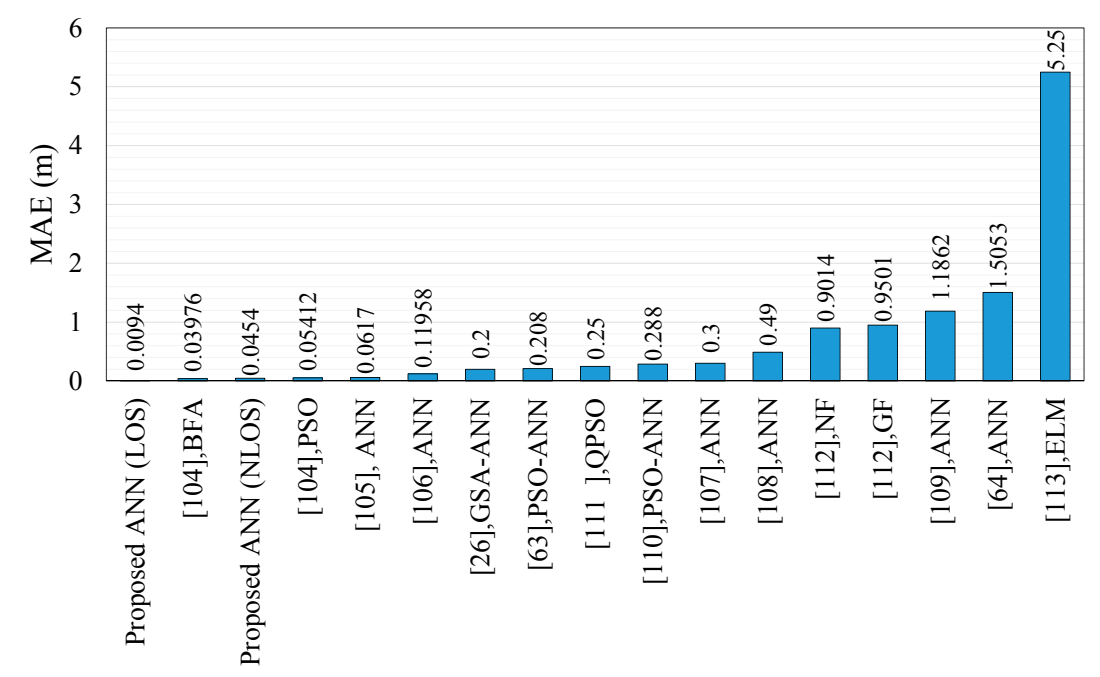

Figure 26. Comparison between MAE of the proposed ANN technique and previous techniques.

\subsection{Power Consumption Comparison}

The proposed FDS that uses DDA can be compared with state-of-the-art methods to assess the performance of the suggested system. The performance of the presented work in terms of current drain is achieved based on the methodology that has been presented in Sections 3.5 and 4 and this is validated by hardware implementation (Section 3.1) and estimated battery life (Section 5.3) based on current consumption measurements in Section 4. The comparison with other related works was accomplished based on the measurement values of the previous works from the literature. The performance of the average current consumption of previous works is presented in related works (Section 2) and in Figure 27. The previous works are plotted on the $x$-axis, and the estimated battery life of the sensor node is plotted on the $y$-axis, as shown in Figure 27. For a fair comparison between the proposed FDS and previous works $[24,50,68,71,87,90,115-121]$, the battery life of the proposed systems in the previous works was recalculated based on $1000 \mathrm{mAh}$. Most of these works are similar to our proposed system because they use different fall detection algorithms to monitor elderly fall events or activities. These articles used different wireless protocols such as Zigbee, WiFi, Bluetooth, and GSM modems to transmit elderly fall events or human activities from the sensor node (worn by the elderly subject) to the sink node. Clearly, the proposed FDS based on DDA outperforms previous systems in terms of battery life, which is extended to 1480 h (62 days), as shown in Figure 27. 


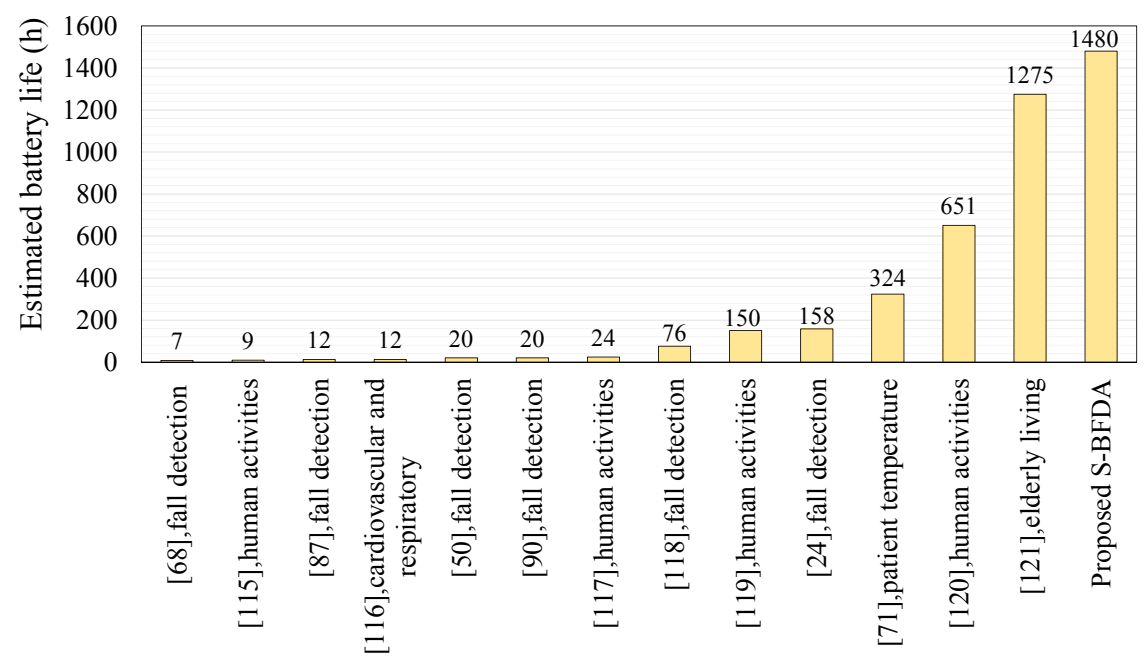

Figure 27. Comparison between battery life of the proposed S-BFDA and previous system.

\section{Conclusions}

This paper addresses three problems related to elderly fall detection, elderly fall localization, and sensor node power consumption. With the use of S-BFDA, an elderly fall is detected with high accuracy in LOS and NLOS environments. The elderly fall is detected by utilizing a combination of accelerometer, tilt, and shock sensors to avoid false detection. An accelerometer sensor is used to measure the inclination angle of the elderly subject during a fall. Most researchers prefer to use the accelerometer sensor because of its small size, high accuracy, and low power consumption. Moreover, it can measure the acceleration in three linear degrees of freedom, namely, $x, y$, and $z$. As a result, the proposed S-BFDA achieves $100 \%$ and $92.5 \%$ fall detection accuracy for LOS and NLOS environments, respectively. The fall detection accuracy in NLOS may increase or decrease depending on the barriers in the tested area. The ANN technique is proposed to localize the elderly fall while the subject is moving in test areas such as a hospital or house. The performance of ANN is explored in terms of MAE, where the ANN technique achieves minimum localization error.

The MAE was 0.0094 and $0.0454 \mathrm{~m}$ for LOS and NLOS, respectively, thereby indicating that the MAE in LOS is approximately five times better than that in NLOS environments. The FDS has limited energy resources, and its battery can be exhausted in a short time. To address this problem, an energy-efficient technique (i.e., DDA) is proposed in this paper. The proposed DDA significantly minimized the power consumption and prolonged the battery life of the FDS. The FDS can work for 62 days without needing to replenish the battery unlike in existing implementations (i.e., without DDA, where the FDS can operate for one day only). Finally, the proposed S-BFDA, ANN technique, and DDA outperform previous systems in terms of accuracy, MAE, and battery life. The results indicate that the proposed system is reliable and can be used in fall detection and localization of elderly people and patients that need special care in indoor environments. For future work, several techniques can be suggested to further improve the power consumption and prolong the battery life of the FDS, as well as minimize the localization error, especially for indoor NLOS environments. The changing of patient location from one place to another can provide a future opportunity to continue the current study to further understand and capture the relevant challenges of the patient's location, especially in practical applications.

Author Contributions: Conceptualization, S.K.G., A.A.-N., and A.M.J.; Methodology, all authors; Software, A.A.-N. and M.J.A.-A.; Validation, S.K.G. and A.A.-N.; Formal Analysis, S.K.G., S.L.M., and M.J.A.-A.; Investigation, S.K.G. and H.M.J.; Resources, A.A.-N. and H.M.J.; Data curation, S.L.M. and A.M.J.; Writing-Original Draft Preparation, S.K.G. and A.M.J.; Writing-Review \& Editing, A.A.-N., S.L.M., M.J.A.-A., and J.C.; Visualization, S.L.M. and H.M.J.; Supervision, S.K.G. and M.J.A.-A.

Funding: This research received no external funding. 
Acknowledgments: The author would like to thank the staff of the Department of Medical Instrumentation Techniques Engineering, Electrical Engineering Technical College, Middle Technical University (MTU), for their support during this study. Authors would like to thanks Al-Rafidain University College for their generosity in financial assistance.

Conflicts of Interest: The authors declare no conflict of interest.

\section{References}

1. Amin, M.G.; Zhang, Y.D.; Ahmad, F.; Ho, K.D. Radar signal processing for elderly fall detection: The future for in-home monitoring. IEEE Signal Process. Mag. 2016, 33, 71-80. [CrossRef]

2. Shinmoto Torres, R.L.; Visvanathan, R.; Hoskins, S.; van den Hengel, A.; Ranasinghe, D.C. Effectiveness of a batteryless and wireless wearable sensor system for identifying bed and chair exits in healthy older people. Sensors 2016, 16, 546. [CrossRef] [PubMed]

3. Chen, M.-C.; Chiu, Y.-W.; Chen, C.-H.; Chen, E.-J. Implementation of fall detection and localized caring system. Math. Probl. Eng. 2013, 2013, 217286. [CrossRef]

4. Naranjo, P.G.V.; Shojafar, M.; Mostafaei, H.; Pooranian, Z.; Baccarelli, E. P-sep: A prolong stable election routing algorithm for energy-limited heterogeneous fog-supported wireless sensor networks. J. Supercomput. 2017, 73, 733-755. [CrossRef]

5. Saleh, N.; Kassem, A.; Haidar, A.M. Energy-efficient architecture for wireless sensor networks in healthcare applications. IEEE Access 2018, 6, 6478-6486. [CrossRef]

6. Naranjo, P.G.V.; Pooranian, Z.; Shojafar, M.; Conti, M.; Buyya, R. Focan: A fog-supported smart city network architecture for management of applications in the internet of everything environments. J. Parallel Distrib. Comput. 2018. [CrossRef]

7. Shojafar, M.; Pooranian, Z.; Naranjo, P.G.V.; Baccarelli, E. Flaps: Bandwidth and delay-efficient distributed data searching in fog-supported p2p content delivery networks. J. Supercomput. 2017, 73, 5239-5260. [CrossRef]

8. Yang, L.; Ren, Y.; Zhang, W. 3D depth image analysis for indoor fall detection of elderly people. Digit. Commun. Netw. 2016, 2, 24-34. [CrossRef]

9. Yang, L.; Ren, Y.; Hu, H.; Tian, B. New fast fall detection method based on spatio-temporal context tracking of head by using depth images. Sensors 2015, 15, 23004. [CrossRef] [PubMed]

10. Diraco, G.; Leone, A.; Siciliano, P. An active vision system for fall detection and posture recognition in elderly healthcare. In Proceedings of the Conference on Design, Automation and Test in Europe, Dresden, Germany, 8-12 March 2010; European Design and Automation Association: Leuven, Belgium, 2010; pp. 1536-1541.

11. Delahoz, Y.; Labrador, M. Survey on fall detection and fall prevention using wearable and external sensors. Sensors 2014, 14, 19806-19842. [CrossRef] [PubMed]

12. Mubashir, M.; Shao, L.; Seed, L. A survey on fall detection: Principles and approaches. Neurocomputing 2013, 100, 144-152. [CrossRef]

13. Sorvala, A.; Alasaarela, E.; Sorvoja, H.; Myllylä, R. A two-threshold fall detection algorithm for reducing false alarms. In Proceedings of the IEEE 6th International Symposium on Medical Information and Communication Technology (ISMICT), La Jolla, CA, USA, 25-29 March 2012; pp. 1-4.

14. Xu, Y.; Zhou, J.; Zhang, P. Rss-based source localization when path-loss model parameters are unknown. IEEE Commun. Lett. 2014, 18, 1055-1058. [CrossRef]

15. Halder, S.J.; Giri, P.; Kim, W. Advanced smoothing approach of RSSI and LQI for indoor localization system. Int. J. Distrib. Sens. Netw. 2015, 11. [CrossRef]

16. Mi, Q.; Stankovic, J.A.; Stoleru, R. Practical and secure localization and key distribution for wireless sensor networks. Ad Hoc Netw. 2012, 10, 946-961. [CrossRef]

17. Liu, Y.; Hu, Y.H.; Pan, Q. Distributed, robust acoustic source localization in a wireless sensor network. IEEE Trans. Signal Process. 2012, 60, 4350-4359. [CrossRef]

18. López, J.D.; Ocampo, C.; Sucerquia, A.; Vargas-Bonilla, J.F. Analyzing multiple accelerometer configurations to detect falls and motion. In Proceedings of the VII Latin American Congress on Biomedical Engineering CLAIB 2016, Bucaramanga, Santander, Colombia, 26-28 October 2016; Torres, I., Bustamante, J., Sierra, D.A., Eds.; Springer: Singapore, 2017; pp. 169-172.

19. Pal, A. Localization algorithms in wireless sensor networks: Current approaches and future challenges. Netw. Protoc. Algorithms 2010, 2, 45-73. [CrossRef] 
20. El Assaf, A.; Zaidi, S.; Affes, S.; Kandil, N. Robust anns-based wsn localization in the presence of anisotropic signal attenuation. IEEE Wirel. Commun. Lett. 2016, 5, 504-507. [CrossRef]

21. Gharghan, S.K.; Nordin, R.; Ismail, M. Energy efficiency of ultra-low-power bicycle wireless sensor networks based on a combination of power reduction techniques. J. Sens. 2016, 2016, 7314207. [CrossRef]

22. Wang, C.; Lu, W.; Narayanan, M.R.; Redmond, S.J.; Lovell, N.H. Low-power technologies for wearable telecare and telehealth systems: A review. Biomed. Eng. Lett. 2015, 5, 1-9. [CrossRef]

23. Panhwar, M.; Shah, S.M.S.; Shah, S.M.Z.S.; Shah, S.M.A.; Chowdhry, B.S. Smart phone based fall detection using auto regression modeling in a non-restrictive setting. Indian J. Sci. Technol. 2017, 10. [CrossRef]

24. Benocci, M.; Tacconi, C.; Farella, E.; Benini, L.; Chiari, L.; Vanzago, L. Accelerometer-based fall detection using optimized zigbee data streaming. Microelectron. J. 2010, 41, 703-710. [CrossRef]

25. Wang, L.-H.; Hsiao, Y.-M.; Xie, X.-Q.; Lee, S.-Y. An outdoor intelligent healthcare monitoring device for the elderly. IEEE Trans. Consum. Electron. 2016, 62, 128-135. [CrossRef]

26. Gharghan, S.K.; Nordin, R.; Ismail, M. A wireless sensor network with soft computing localization techniques for track cycling applications. Sensors 2016, 16, 1043. [CrossRef] [PubMed]

27. Cheng, J.; Chen, X.; Shen, M. A framework for daily activity monitoring and fall detection based on surface electromyography and accelerometer signals. IEEE J. Biomed. Health Inform. 2013, 17, 38-45. [CrossRef] [PubMed]

28. Chen, S.-K.; Kao, T.; Chan, C.-T.; Huang, C.-N.; Chiang, C.-Y.; Lai, C.-Y.; Tung, T.-H.; Wang, P.-C. A reliable transmission protocol for zigbee-based wireless patient monitoring. IEEE Trans. Inf. Technol. Biomed. 2012, 16, 6-16. [CrossRef] [PubMed]

29. Özdemir, A.T.; Barshan, B. Detecting falls with wearable sensors using machine learning techniques. Sensors 2014, 14, 10691-10708. [CrossRef] [PubMed]

30. Felisberto, F.; Laza, R.; Fdez-Riverola, F.; Pereira, A. A distributed multiagent system architecture for body area networks applied to healthcare monitoring. BioMed Res. Int. 2015, 2015, 192454. [CrossRef] [PubMed]

31. Rescio, G.; Leone, A.; Siciliano, P. Supervised expert system for wearable mems accelerometer-based fall detector. J. Sens. 2013, 2013, 254629. [CrossRef]

32. Lim, D.; Park, C.; Kim, N.H.; Kim, S.-H.; Yu, Y.S. Fall-detection algorithm using 3-axis acceleration: Combination with simple threshold and hidden markov model. J. Appl. Math. 2014, 2014, 896030. [CrossRef]

33. Özdemir, A.T. An analysis on sensor locations of the human body for wearable fall detection devices: Principles and practice. Sensors 2016, 16, 1161. [CrossRef] [PubMed]

34. Charlon, Y.; Fourty, N.; Bourennane, W.; Campo, E. Design and evaluation of a device worn for fall detection and localization: Application for the continuous monitoring of risks incurred by dependents in an alzheimer's care unit. Expert Syst. Appl. 2013, 40, 7316-7330. [CrossRef]

35. He, J.; Hu, C.; Wang, X. A smart device enabled system for autonomous fall detection and alert. Int. J. Distrib. Sens. Netw. 2016, 2016. [CrossRef]

36. Panicker, N.V.; Kumar, A.S. Tablet pc enabled body sensor system for rural telehealth applications. Int. J. Telemed. Appl. 2016, 2016. [CrossRef] [PubMed]

37. Oporto, E.; Vilcahuaman, L. Fall detection system for elderly by MEMS accelerometer and SMS alert. In Proceedings of the VII Latin American Congress on Biomedical Engineering CLAIB 2016, Bucaramanga, Santander, Colombia, 26-28 October 2016; Springer: Singapore, 2017; pp. 114-117.

38. Huynh, Q.T.; Nguyen, U.D.; Irazabal, L.B.; Ghassemian, N.; Tran, B.Q. Optimization of an accelerometer and gyroscope-based fall detection algorithm. J. Sens. 2015, 2015, 452078. [CrossRef]

39. Majumder, A. Development of a Wireless Mobile Computing Platform for Fall Risk Prediction. Ph.D. Thesis, Marquette University, Milwaukee, WI, USA, 2016.

40. Luque, R.; Casilari, E.; Morón, M.-J.; Redondo, G. Comparison and characterization of android-based fall detection systems. Sensors 2014, 14, 18543-18574. [CrossRef] [PubMed]

41. Zhao, Z.; Chen, Y.; Liu, J. Fall detecting and alarming based on mobile phone. In Proceedings of the 7th International Conference on Ubiquitous Intelligence \& Computing and 7th International Conference on Autonomic \& Trusted Computing (UIC/ATC), Xi'an, China, 26-29 October 2010; pp. 494-497.

42. Zhao, Z.; Chen, Y.; Wang, S.; Chen, Z. Fallalarm: Smart phone based fall detecting and positioning system. Procedia Comput. Sci. 2012, 10, 617-624. [CrossRef]

43. Majumder, A.J.A.; Zerin, I.; Ahamed, S.I.; Smith, R.O. A multi-sensor approach for fall risk prediction and prevention in elderly. ACM SIGAPP Appl. Comput. Rev. 2014, 14, 41-52. [CrossRef] 
44. Dai, J.; Bai, X.; Yang, Z.; Shen, Z.; Xuan, D. Mobile phone-based pervasive fall detection. Pers. Ubiquitous Comput. 2010, 14, 633-643. [CrossRef]

45. Habib, M.A.; Mohktar, M.S.; Kamaruzzaman, S.B.; Lim, K.S.; Pin, T.M.; Ibrahim, F. Smartphone-based solutions for fall detection and prevention: Challenges and open issues. Sensors 2014, 14, 7181-7208. [CrossRef] [PubMed]

46. Mellone, S.; Tacconi, C.; Schwickert, L.; Klenk, J.; Becker, C.; Chiari, L. Smartphone-based solutions for fall detection and prevention: The farseeing approach. Z. Gerontol. Geriatr. 2012, 45, 722-727. [CrossRef] [PubMed]

47. Abbate, S.; Avvenuti, M.; Bonatesta, F.; Cola, G.; Corsini, P.; Vecchio, A. A smartphone-based fall detection system. Pervasive Mob. Comput. 2012, 8, 883-899. [CrossRef]

48. Medrano, C.; Plaza, I.; Igual, R.; Sánchez, Á.; Castro, M. The effect of personalization on smartphone-based fall detectors. Sensors 2016, 16, 117. [CrossRef] [PubMed]

49. Huang, C.-N.; Chan, C.-T. Zigbee-based indoor location system by k-nearest neighbor algorithm with weighted rssi. Procedia Comput. Sci. 2011, 5, 58-65. [CrossRef]

50. Wu, F.; Zhao, H.; Zhao, Y.; Zhong, H. Development of a wearable-sensor-based fall detection system. Int. J. Telemed. Appl. 2015, 2015. [CrossRef] [PubMed]

51. Bian, Z.-P.; Hou, J.; Chau, L.-P.; Magnenat-Thalmann, N. Fall detection based on body part tracking using a depth camera. IEEE J. Biomed. Health Inform. 2015, 19, 430-439. [CrossRef] [PubMed]

52. Yu, M.; Yu, Y.; Rhuma, A.; Naqvi, S.M.R.; Wang, L.; Chambers, J.A. An online one class support vector machine-based person-specific fall detection system for monitoring an elderly individual in a room environment. IEEE J. Biomed. Health Inform. 2013, 17, 1002-1014. [PubMed]

53. Ozcan, K.; Mahabalagiri, A.K.; Casares, M.; Velipasalar, S. Automatic fall detection and activity classification by a wearable embedded smart camera. IEEE J. Emerg. Sel. Top. Circuits Syst. 2013, 3, 125-136. [CrossRef]

54. Su, B.Y.; Ho, K.; Rantz, M.J.; Skubic, M. Doppler radar fall activity detection using the wavelet transform. IEEE Trans. Biomed. Eng. 2015, 62, 865-875. [CrossRef] [PubMed]

55. Sachs, J.; Herrmann, R. M-sequence-based ultra-wideband sensor network for vitality monitoring of elders at home. IET Radar Sonar Navig. 2015, 9, 125-137. [CrossRef]

56. Garripoli, C.; Mercuri, M.; Karsmakers, P.; Soh, P.J.; Crupi, G.; Vandenbosch, G.A.; Pace, C.; Leroux, P.; Schreurs, D. Embedded dsp-based telehealth radar system for remote in-door fall detection. IEEE J. Biomed. Health Inform. 2015, 19, 92-101. [CrossRef] [PubMed]

57. Fernández-Caballero, A.; Sokolova, M.V.; Serrano-Cuerda, J. Lateral inhibition in accumulative computation and fuzzy sets for human fall pattern recognition in colour and infrared imagery. Sci. World J. 2013, 2013, 935026. [CrossRef] [PubMed]

58. Ndzi, D.L.; Harun, A.; Ramli, F.M.; Kamarudin, M.L.; Zakaria, A.; Shakaff, A.Y.M.; Jaafar, M.N.; Zhou, S.; Farook, R.S. Wireless sensor network coverage measurement and planning in mixed crop farming. Comput. Electron. Agric. 2014, 105, 83-94. [CrossRef]

59. Jawad, H.M.; Nordin, R.; Gharghan, S.K.; Jawad, A.M.; Ismail, M. Energy-efficient wireless sensor networks for precision agriculture: A review. Sensors 2017, 17, 1781. [CrossRef] [PubMed]

60. Xu, J.; Liu, W.; Lang, F.; Zhang, Y.; Wang, C. Distance measurement model based on RSSI in WSN. Wirel. Sens. Netw. 2010, 2, 606. [CrossRef]

61. Sahu, P.K.; Wu, E.H.-K.; Sahoo, J. Durt: Dual RSSI trend based localization for wireless sensor networks. IEEE Sens. J. 2013, 13, 3115-3123. [CrossRef]

62. Cotuk, H.; Bicakci, K.; Tavli, B.; Uzun, E. The impact of transmission power control strategies on lifetime of wireless sensor networks. IEEE Trans. Comput. 2014, 63, 2866-2879. [CrossRef]

63. Gharghan, S.K.; Nordin, R.; Ismail, M.; Ali, J.A. Accurate wireless sensor localization technique based on hybrid PSO-ANN algorithm for indoor and outdoor track cycling. IEEE Sens. J. 2016, 16, 529-541. [CrossRef]

64. Payal, A.; Rai, C.S.; Reddy, B.R. Analysis of some feedforward artificial neural network training algorithms for developing localization framework in wireless sensor networks. Wirel. Pers. Commun. 2015, 82, 2519-2536. [CrossRef]

65. Kukolj, D.; Levi, E. Identification of complex systems based on neural and takagi-sugeno fuzzy model. IEEE Trans. Syst. Man Cybern. Part B Cybern. 2004, 34, 272-282. [CrossRef]

66. Kumar, S.; Jeon, S.M.; Lee, S.R. Localization estimation using artificial intelligence technique in wireless sensor networks. J. Korea Inf. Commun. Soc. 2014, 39, 820-827. 
67. Aboelela, E.H.; Khan, A.H. Wireless sensors and neural networks for intruders detection and classification. In Proceedings of the International Conference on Information Networking (ICOIN), Bali, Indonesia, 1-3 February 2012; pp. 138-143.

68. He, J.; Bai, S.; Wang, X. An unobtrusive fall detection and alerting system based on kalman filter and bayes network classifier. Sensors 2017, 17, 1393. [CrossRef] [PubMed]

69. Wang, C.; Lu, W.; Narayanan, M.R.; Chang, D.C.W.; Lord, S.R.; Redmond, S.J.; Lovell, N.H. Low-power fall detector using triaxial accelerometry and barometric pressure sensing. IEEE Trans. Ind. Inform. 2016, 12, 2302-2311. [CrossRef]

70. Gharghan, S.K.; Nordin, R.; Ismail, M. An ultra-low power wireless sensor network for bicycle torque performance measurements. Sensors 2015, 15, 11741. [CrossRef] [PubMed]

71. Gharghan, S.K. Energy-efficient remote temperature monitoring system for patients based on GSM modem and microcontroller. J. Commun. 2017, 12, 433-442. [CrossRef]

72. Data Acquisition and Graphical Plotting Software for Microcontrolles (Makerplot). Available online: http: / / www.makerplot.com (accessed on 3 March 2014).

73. Aziz, O.; Klenk, J.; Schwickert, L.; Chiari, L.; Becker, C.; Park, E.J.; Mori, G.; Robinovitch, S.N. Validation of accuracy of SVM-based fall detection system using real-world fall and non-fall datasets. PLoS ONE 2017, 12, e0180318. [CrossRef] [PubMed]

74. Wang, H.; Zhang, D.; Wang, Y.; Ma, J.; Wang, Y.; Li, S. Rt-fall: A real-time and contactless fall detection system with commodity wifi devices. IEEE Trans. Mob. Comput. 2017, 16, 511-526. [CrossRef]

75. Planinc, R.; Kampel, M. Robust fall detection by combining 3D data and fuzzy logic. In Computer Vision-ACCV 2012 Workshops: ACCV 2012 International Workshops, Daejeon, Korea, 5-6 November 2012, Revised Selected Papers, Part II; Park, J.-I., Kim, J., Eds.; Springer: Berlin/Heidelberg, Germany, 2013; pp. 121-132.

76. Huang, C.-N.; Chan, C.-T. A zigbee-based location-aware fall detection system for improving elderly telecare. Int. J. Environ. Res. Public Health 2014, 11, 4233-4248. [CrossRef] [PubMed]

77. Zhang, S.; McCullagh, P.; Zhang, J.; Yu, T. A smartphone based real-time daily activity monitoring system. Clust. Comput. 2014, 17, 711-721. [CrossRef]

78. Zhao, K.; Jia, K.; Liu, P. Fall detection algorithm based on human posture recognition. In Advances in Intelligent Information Hiding and Multimedia Signal Processing: Proceeding of the Twelfth International Conference on Intelligent Information Hiding and Multimedia Signal Processing, Nov., 21-23, 2016, Kaohsiung, Taiwan, Volume 2; Pan, J.-S., Tsai, P.-W., Huang, H.-C., Eds.; Springer International Publishing: Cham, Switzerland, 2017; pp. 119-126.

79. Chen, K.-H.; Yang, J.-J.; Jaw, F.-S. Accelerometer-based fall detection using feature extraction and support vector machine algorithms. Instrum. Sci. Technol. 2016, 44, 333-342. [CrossRef]

80. Kwolek, B.; Kepski, M. Fuzzy inference-based fall detection using kinect and body-worn accelerometer. Appl. Soft Comput. 2016, 40, 305-318. [CrossRef]

81. Gibson, R.M.; Amira, A.; Ramzan, N.; Casaseca-de-la-Higuera, P.; Pervez, Z. Multiple comparator classifier framework for accelerometer-based fall detection and diagnostic. Appl. Soft Comput. 2016, 39, 94-103. [CrossRef]

82. Fang, Y.-C.; Dzeng, R.-J. Accelerometer-based fall-portent detection algorithm for construction tiling operation. Autom. Constr. 2017, 84, 214-230. [CrossRef]

83. Wang, C.; Lee, S.; Ho, J.; Na, Y.; Min, S.D. Detection of optimal activity recognition algorithm for elderly using smartphone. In Advances in Computer Science and Ubiquitous Computing: CSA-CUTE2016; Park, J.J., Pan, Y., Yi, G., Loia, V., Eds.; Springer: Singapore, 2017; pp. 1013-1018.

84. Hakim, A.; Huq, M.S.; Shanta, S.; Ibrahim, B.S.K.K. Smartphone based data mining for fall detection: Analysis and design. Procedia Comput. Sci. 2017, 105, 46-51. [CrossRef]

85. Veeraputhiran, A.; Sankararajan, R. Feature based fall detection system for elders using compressed sensing in WVSN. Wirel. Netw. 2017. [CrossRef]

86. Erol, B.; Amin, M.G.; Boashash, B. Range-doppler radar sensor fusion for fall detection. In Proceedings of the IEEE Radar Conference (RadarConf), Seattle, WA, USA, 8-12 May 2017; pp. 819-824.

87. Ngu, A.; Wu, Y.; Zare, H.; Polican, A.; Yarbrough, B.; Yao, L. Fall detection using smartwatch sensor data with accessor architecture. In Smart Health: International Conference, ICSH 2017, Hong Kong, China, June 26-27, 2017, Proceedings; Chen, H., Zeng, D.D., Karahanna, E., Bardhan, I., Eds.; Springer International Publishing: Cham, Switzerland, 2017; pp. 81-93. 
88. Alazrai, R.; Momani, M.; Daoud, M. Fall detection for elderly from partially observed depth-map video sequences based on view-invariant human activity representation. Appl. Sci. 2017, 7, 316. [CrossRef]

89. Ozcan, K.; Velipasalar, S.; Varshney, P.K. Autonomous fall detection with wearable cameras by using relative entropy distance measure. IEEE Trans. Hum.-Mach. Syst. 2017, 47, 31-39. [CrossRef]

90. Di Rosa, M.; Hausdorff, J.M.; Stara, V.; Rossi, L.; Glynn, L.; Casey, M.; Burkard, S.; Cherubini, A. Concurrent validation of an index to estimate fall risk in community dwelling seniors through a wireless sensor insole system: A pilot study. Gait Posture 2017, 55, 6-11. [CrossRef] [PubMed]

91. Nizam, Y.; Mohd, M.N.H.; Jamil, M.M.A. Human fall detection from depth images using position and velocity of subject. Procedia Comput. Sci. 2017, 105, 131-137. [CrossRef]

92. Wang, Y.; Wu, K.; Ni, L.M. Wifall: Device-free fall detection by wireless networks. IEEE Trans. Mob. Comput. 2017, 16, 581-594. [CrossRef]

93. Chen, K.-H.; Hsu, Y.-W.; Yang, J.-J.; Jaw, F.-S. Enhanced characterization of an accelerometer-based fall detection algorithm using a repository. Instrum. Sci. Technol. 2017, 45, 382-391. [CrossRef]

94. Ismail, M.M.B.; Bchir, O. Automatic fall detection using membership based histogram descriptors. J. Comput. Sci. Technol. 2017, 32, 356-367. [CrossRef]

95. Liu, H.; Guo, Y. A vision-based fall detection algorithm of human in indoor environment. In Proceedings of the Second International Conference on Photonics and Optical Engineering, Xi'an, China, 14-17 October 2016; SPIE: Bellingham, WA, USA, 2017; p. 6.

96. Álvarez de la Concepción, M.Á.; Soria Morillo, L.M.; Álvarez García, J.A.; González-Abril, L. Mobile activity recognition and fall detection system for elderly people using ameva algorithm. Pervasive Mob. Comput. 2017, 34, 3-13. [CrossRef]

97. de Quadros, T.; Lazzaretti, A.E.; Schneider, F.K. Development and evaluation of a method for fall detection based on a wrist-located device. In Proceedings of the VII Latin American Congress on Biomedical Engineering CLAIB 2016, Bucaramanga, Santander, Colombia, 26-28 October 2016; Torres, I., Bustamante, J., Sierra, D.A., Eds.; Springer: Singapore, 2017; pp. 256-259.

98. Sucerquia, A.; López, J.; Vargas-Bonilla, J. Sisfall: A fall and movement dataset. Sensors 2017, 17, 198. [CrossRef] [PubMed]

99. Zhu, L.; Wang, R.; Wang, Z.; Yang, H. Tagcare: Using rfids to monitor the status of the elderly living alone. IEEE Access 2017, 5, 11364-11373. [CrossRef]

100. Aziz, O.; Musngi, M.; Park, E.J.; Mori, G.; Robinovitch, S.N. A comparison of accuracy of fall detection algorithms (threshold-based vs. Machine learning) using waist-mounted tri-axial accelerometer signals from a comprehensive set of falls and non-fall trials. Med. Biol. Eng. Comput. 2017, 55, 45-55. [CrossRef] [PubMed]

101. Kianoush, S.; Savazzi, S.; Vicentini, F.; Rampa, V.; Giussani, M. Device-free rf human body fall detection and localization in industrial workplaces. IEEE Internet Things J. 2017, 4, 351-362. [CrossRef]

102. Li, Y.; Zhai, Q.; Ding, S.; Yang, F.; Li, G.; Zheng, Y.F. Efficient health-related abnormal behavior detection with visual and inertial sensor integration. Pattern Anal. Appl. 2017. [CrossRef]

103. Hsieh, C.-Y.; Liu, K.-C.; Huang, C.-N.; Chu, W.-C.; Chan, C.-T. Novel hierarchical fall detection algorithm using a multiphase fall model. Sensors 2017, 17, 307. [CrossRef] [PubMed]

104. Kulkarni, R.V.; Venayagamoorthy, G.K. Bio-inspired algorithms for autonomous deployment and localization of sensor nodes. IEEE Trans. Syst. Man Cybern. Part C (Appl. Rev.) 2010, 40, 663-675. [CrossRef]

105. Li, S.; Qin, F. A dynamic neural network approach for solving nonlinear inequalities defined on a graph and its application to distributed, routing-free, range-free localization of wsns. Neurocomputing 2013, 117, 72-80. [CrossRef]

106. Zhao, L.-Z.; Wen, X.-B.; Li, D. Amorphous localization algorithm based on BP artificial neural network. Int. J. Distrib. Sens. Netw. 2015, 11, 9. [CrossRef]

107. Kumar, S.; Sharma, R.; Vans, E. Localization for wireless sensor networks: A neural network approach. Int. J. Comput. Networks Commun. 2016, 8, 61-71. [CrossRef]

108. Payal, A.; Rai, C.; Reddy, B. Comparative analysis of bayesian regularization and levenberg-marquardt training algorithm for localization in wireless sensor network. In Proceedings of the 15th International Conference on Advanced Communication Technology (ICACT), PyeongChang, Korea, 27-30 January 2013; pp. 191-194. 
109. Payal, A.; Rai, C.; Reddy, B. Artificial neural networks for developing localization framework in wireless sensor networks. In Proceedings of the International Conference on Data Mining and Intelligent Computing (ICDMIC), Delhi, India, 5-6 September 2014; pp. 1-6.

110. Jun, W.; Fu, Z.; Tiansi, R.; Xun, C.; Gang, L. A novel hybrid localization method for wireless sensor network. Int. J. Smart Sens. Intell. Syst. 2016, 9, 1323-1340. [CrossRef]

111. Wu, X.; Deng, F.; Chen, Z. Rfid 3D-landmarc localization algorithm based on quantum particle swarm optimization. Electronics 2018, 7, 19.

112. Nekooei, S.M.; Manzuri-Shalmani, M. Location finding in wireless sensor network based on soft computing methods. In Proceedings of the International Conference on Control, Automation and Systems Engineering (CASE), Singapore, 30-31 July 2011; pp. 1-5.

113. So-In, C.; Permpol, S.; Rujirakul, K. Soft computing-based localizations in wireless sensor networks. Pervasive Mob. Comput. 2016, 29, 17-37. [CrossRef]

114. Wang, Z.; Yang, Z.; Dong, T. A review of wearable technologies for elderly care that can accurately track indoor position, recognize physical activities and monitor vital signs in real time. Sensors 2017, $17,341$. [CrossRef] [PubMed]

115. Liang, Y.; Zhou, X.; Yu, Z.; Guo, B. Energy-efficient motion related activity recognition on mobile devices for pervasive healthcare. Mob. Netw. Appl. 2014, 19, 303-317. [CrossRef]

116. Miramontes, R.; Aquino, R.; Flores, A.; Rodríguez, G.; Anguiano, R.; Ríos, A.; Edwards, A. Plaimos: A remote mobile healthcare platform to monitor cardiovascular and respiratory variables. Sensors 2017, 17, 176. [CrossRef] [PubMed]

117. Kantoch, E.; Grochala, D.; Kajor, M. Bio-inspired topology of wearable sensor fusion for telemedical application. In Artificial Intelligence and Soft Computing: 16th International Conference, ICAISC 2017, Zakopane, Poland, June 11-15, 2017, Proceedings, Part I; Rutkowski, L., Korytkowski, M., Scherer, R., Tadeusiewicz, R., Zadeh, L.A., Zurada, J.M., Eds.; Springer International Publishing: Cham, Switzerland, 2017; pp. 658-667.

118. Nguyen Gia, T.; Sarker, V.K.; Tcarenko, I.; Rahmani, A.M.; Westerlund, T.; Liljeberg, P.; Tenhunen, H. Energy efficient wearable sensor node for IoT-based fall detection systems. Microprocess. Microsyst. 2018, 56, 34-46. [CrossRef]

119. Wang, Y.; Krishnamachari, B.; Annavaram, M. Semi-markov state estimation and policy optimization for energy efficient mobile sensing. In Proceedings of the Annual IEEE Communications Society Conference on Sensor, Mesh and Ad Hoc Communications and Networks (SECON), Seoul, Korea, 18-21 June 2012; pp. 533-541.

120. Ren, L.; Zhang, Q.; Shi, W. Low-power fall detection in home-based environments. In Proceedings of the 2nd ACM international workshop on Pervasive Wireless Healthcare, Hilton Head, SC, USA, 11 June 2012; pp. 39-44.

121. Pinto, S.; Cabral, J.; Gomes, T. We-care: An iot-based health care system for elderly people. In Proceedings of the IEEE International Conference on Industrial Technology (ICIT), Toronto, ON, Canada, 22-25 March 2017; pp. 1378-1383.

(c) 2018 by the authors. Licensee MDPI, Basel, Switzerland. This article is an open access article distributed under the terms and conditions of the Creative Commons Attribution (CC BY) license (http:/ / creativecommons.org/licenses/by/4.0/). 